\title{
Feynman amplitudes in periodically compactified spaces: Spin 0
}

\author{
E. Cavalcanti®* \\ Centro Brasileiro de Pesquisas Físicas/MCTI, 22290-180 Rio de Janeiro, RJ, Brazil
}

(Received 1 June 2021; accepted 27 September 2021; published 21 October 2021)

\begin{abstract}
We propose an extension of the Schwinger parametric representation for Feynman amplitudes in $D$ euclidean dimensions to a scenario where $d$ dimensions are compactified $(d<D)$ through the introduction of periodic boundary conditions in space. We obtain two valid representations, one useful near the bulk (large compactification length) and another useful near the dimensional reduction (small compactification length). Also, to illustrate, we exhibit some Feynman amplitudes up to three loops in a compactified scalar field theory.
\end{abstract}

DOI: 10.1103/PhysRevD.104.085019

\section{INTRODUCTION}

One essential task in perturbative quantum field theory is to obtain, for a given theory, the amplitude of graph $G$ at some arbitrary order. This task is decades older and its solution is already textbook material; the most known parametric representations are those of Feynman [1,2], Schwinger [1-3], and Mellin [4,5], although other efforts, like the Complete Mellin representation [6-10], for example, were also studied. The employment of these representations in a general setting employs the Symanzik polynomials, which can be directly extracted from the topology of the graph by inspecting the 1-trees and 2-trees $[2,3,11]$. That way, one can avoid the otherwise cumbersome computation of these polynomials and we have an "easy" prescription to compute higher-order diagrams. This first simple task was essential for the important developments that follow, for example, proving the renormalizability of a quantum field theory up to all orders or extracting the asymptotic behavior of a diagram.

Although this topic, to obtain a parametric representation, is completely settled for theories in noncompact space-time-let us refer to the Euclidean space-time with $D$ dimensions-it has not yet been established for theories in compactified dimensions. If the only interest of someone is the proof of renormalizability, one can justify this lack of understanding concerning theories in compactified dimensions due to the knowledge that the divergent behavior in the amplitudes of compactified theories come from the bulk, that is, the contribution related to the noncompactified

*erich@cbpf.br

Published by the American Physical Society under the terms of the Creative Commons Attribution 4.0 International license. Further distribution of this work must maintain attribution to the author(s) and the published article's title, journal citation, and DOI. Funded by SCOAP ${ }^{3}$. space $[12,13]$. This means that there is no need to prove renormalizability again as it comes directly from the proof in the bulk scenario. However, this does not justify the absence of this exploration in the literature. Right now, there is no established parametric representation to deal with higher-order diagrams in a scenario with compactified spaces neither an asymptotic expansion for this scenario. The first step, if one is interested in some progress in topics that depend on higher-order corrections of Feynman diagrams in compactified spaces, is to establish a useful parametric representation, and this is the purpose of this work.

There are plenty of ways to introduce compactified dimensions, just as there are many possible choices of boundary conditions. Perhaps the most simple scenarios are periodically compactified theories with just one compactified dimension, which is exactly the highly explored scenario of field theory at finite temperature [14-18], where the inverse temperature, $\beta=1 / T$, is introduced as the compactification length of a periodically compactified dimension through the Matsubara formalism of imaginary time. In recent years, there was also a growing interest in field theory with a small circle compactification [19-23], which is somewhat equivalent to finite temperature but allows us to impose antiperiodic or even twisted boundary conditions in the spatial compactification. If one deals with the thermal partition function, the boundary condition in the imaginary time is restricted by the Kubo-MartinSchwinger (KMS) condition [14-18] to follow the bosonic/fermionic nature of the field. We are free of this restriction if we deal with spatial compactification or when we are not interested in thermal partition functions-a wellknown such scenario occurs in supersymmetric models where both bosons and fermions have periodic boundary conditions and one can discuss the existence of a Witten index [24]. When it comes to the extension to more compactified dimensions and different boundary 
conditions, the range of applicability just grows even more: the Casimir effect [25-29], superstring theory [30-32], quantum field theory with an small extradimension [33-41], finite volume considerations in quantum field theory (QFT) models and in particle physics [42-55], and so on. To see more applications we refer to the works $[18,29,56,57]$ and references therein.

In the last decades, Refs. [18,56] established the so-called "quantum field theory in toroidal topologies," a formalism to deal with periodically compactified dimensions. This is born as an extension of the Matsubara prescription of imaginary time to more dimensions, producing a topology $\Gamma_{D}^{d}=\mathbb{R}^{D-d} \times \mathbb{S}_{L_{1}} \times \cdots \times \mathbb{S}_{L_{d}}$, where $\mathbb{S}_{L_{i}}$ represents each of the $d$ compactifications that can be imposed by periodic/antiperiodic boundary conditions, ${ }^{1}$ with $L_{i}$ as the characteristic compactification length. Within this formulation, many applications were explored both in the field of particle physics and in the field of condensed matter [59-71]. In recent years, a somewhat similar approach [57] also started to explore the formulation and applications of periodically compactified QFT.

So far, only few attempts were done [72-75] in the direction to establish a parametric representation for Feynman amplitudes in compactified spaces. Three decades ago, Benhamou [72,73] proposed a parametric representation for field theory at a finite temperature that recovers the Symanzik polynomials for the compactified part and that has as the zero-temperature limit the usual noncompactified parametric representation. One decade ago there was an attempt [74] to build for scalar field models some representation for the scenario of periodically compactified dimensions. Also, a recent work (Ref. [75]) deals with the parametric representation for fields with different spins in a compactified space.

In Sec. II we start building the Schwinger parametric representation for a graph in periodically compactified spaces. We see that there are two possible paths to follow: 1) we can consider the scale where the lengths of the compactified dimensions are very small (Sec. III), meaning that we are close to a dimensional reduction; 2) we can assume that the lengths are very large (Sec. IV), so we are close to the bulk scenario without compactifications. Both paths are equivalent and one could transport from one to another [76,77], but each one of them is more useful in one regime (near the dimensional reduction or the bulk), due to quicker convergence. After this, we illustrate the representation by showing some diagrams in Sec. V with the useful information to write their parametric representation. In the conclusions, we indicate some further developments.

\footnotetext{
${ }^{1}$ One can easily extend it to quasiperiodic (also called twisted or anyonic) boundary conditions [58].
}

\section{PARAMETRIC REPRESENTATION OF COMPACTIFIED FEYNMAN DIAGRAMS}

Let us start by considering a general scalar scenario [1,2],

$$
\begin{aligned}
\tilde{\mathcal{I}}_{G}= & C_{G} \prod_{i=1}^{I}\left[\int \frac{d^{D} K_{i}}{(2 \pi)^{D}} \frac{1}{K_{i}^{2}+m_{i}^{2}}\right] \\
& \times \prod_{v=1}^{V}\left[(2 \pi)^{D} \delta^{D}\left(P_{v}-\sum_{i} \varepsilon_{v i} K_{i}\right)\right],
\end{aligned}
$$

where $D$ is the number of dimensions (we consider an Euclidian space-time), $C_{G}$ is a factor related to the vertices and the symmetry of the graph, $K_{i}$ are the internal momenta-that run from $i=1, \ldots, I$, where $I$ is the number of internal lines, $P_{v}$ are the sum of external momenta coming into the vertex $v$-that runs from $v=1, \ldots, V$, where $V$ is the number of vertices, and $\varepsilon_{v i}$ is the incidence matrix $\left(\varepsilon_{v i}=+1\right.$ if the line $i$ starts at the vertex $v, \varepsilon_{v i}=-1$ if the line $i$ ends at the vertex $v, m_{i}$ is the mass term associated with each line, and $\varepsilon_{v i}=0$ if the line $i$ is not related to the vertex $v$ ). To abbreviate notation, $\prod_{i}$ will always refer to $\prod_{i=1}^{I}$, unless otherwise specified.

We introduce $d(d<D)$ periodically compactified dimensions by employing an extension of the Matsubara formalism of imaginary time $[18,56]$. The prescription is well established to also consider the introduction of a chemical potential or to employ quasiperiodic/anyonic boundary conditions. However, for this work, we stick with just the periodic boundary conditions. We denote by a capital letter the full $D$-dimensional momenta $\left(K_{i}\right)$, and by lower case the momenta related to the $D-d$ noncompactified dimensions, that is

$$
\begin{aligned}
K_{i}^{2} & \rightarrow k_{i}^{2}+\sum_{\alpha}\left(\omega_{\alpha}^{k_{i}}\right)^{2}, \\
\int \frac{d^{D} K_{i}}{(2 \pi)^{D}} & \rightarrow \int \frac{d^{D-d} k_{i}}{(2 \pi)^{D-d}} \frac{1}{\prod_{\alpha} L_{\alpha}} \sum_{\substack{n_{\alpha}^{k_{i}} \in \mathbb{Z} \\
\forall \alpha}}, \\
\omega_{\alpha}^{k_{i}} & =\frac{2 \pi}{L_{\alpha}} n_{\alpha}^{k_{i}},
\end{aligned}
$$

where the index $\alpha$ runs over the $d$ compactified dimensions, $L_{\alpha}$ is the characteristic length for each of them and $\omega_{\alpha}^{k_{i}}$ is the $\alpha$-th Matsubara frequency associated with the momenta $k_{i}$. Notice that $\prod_{\alpha}$ must be understood as $\prod_{\alpha=1}^{d}$.

Therefore, the amplitude for the graph $G$ in compactified dimensions turns to be 
$\tilde{\mathcal{I}}_{G}=C_{G} \prod_{i}\left[\int \frac{d^{D-d} k_{i}}{(2 \pi)^{D-d}} \frac{1}{\prod_{\alpha} L_{\alpha}} \sum_{\substack{k_{i} \in \mathbb{Z} \\ n_{i}}} \frac{1}{k_{i}^{2}+\sum_{\alpha}\left(\omega_{\alpha}^{k_{i}}\right)^{2}+m_{i}^{2}}\right] \times \prod_{v=1}^{V}\left[(2 \pi)^{D} \delta^{D-d}\left(p_{v}-\sum_{i} \varepsilon_{v i} k_{i}\right) \prod_{\alpha} \delta\left(\omega_{\alpha}^{p_{v}}-\sum_{i} \varepsilon_{v i} \omega_{\alpha}^{k_{i}}\right)\right]$.

To proceed, we start dealing with the noncompactified dimensions. At this point the procedure is closely related to the standard one, but is exhibited for completeness and clarity. We introduce an integral representation for the propagator and the conservation deltas, introducing the Schwinger parameters $u_{i}$ related to each internal line,

$$
\begin{aligned}
\tilde{\mathcal{I}}_{G}= & C_{G} \prod_{i}\left[\int \frac{d^{D-d} k_{i}}{(2 \pi)^{D-d}} \frac{1}{\prod_{\alpha} L_{\alpha}} \sum_{\substack{k_{i} k_{i} \in \mathbb{Z} \\
\forall \forall}} \int_{0}^{\infty} d u_{i} e^{-u_{i}\left[k_{i}^{2}+\sum_{\alpha}\left(\omega_{\alpha}^{k_{i}}\right)^{2}+m_{i}^{2}\right]}\right] \\
& \times \prod_{v=1}^{V}\left[\int d^{D-d} y_{v} e^{-i y_{v} \cdot\left(p_{v}-\sum_{i} \varepsilon_{v i} k_{i}\right)} \prod_{\alpha}\left[\int d z_{v}^{(\alpha)} e^{-i z_{v}^{(\alpha)}\left(\omega_{\alpha}^{p_{v}}-\sum_{i} \varepsilon_{v i} \omega_{\alpha}^{k_{i}}\right)}\right]\right] .
\end{aligned}
$$

We can interchange the sign of integrals and sums to make evident the integral over the internal momenta $k_{i}$ and also the sum over the internal frequencies $\omega_{\alpha}^{k_{i}}$, that is

$$
\begin{aligned}
& \tilde{\mathcal{I}}_{G}=C_{G}\left[\prod_{i} \int_{0}^{\infty} d u_{i}\right]\left[\prod_{v=1}^{V} \int d^{D-d} y_{v}\right]\left[\prod_{v=1}^{V} \prod_{\alpha} \int d z_{v}^{(\alpha)}\right] e^{-\sum_{i} u_{i} m_{i}^{2}} e^{-i \sum_{v=1}^{V} \sum_{\alpha} z_{v}^{(\alpha)} \omega_{\alpha}^{p_{v}}} e^{-i \sum_{v=1}^{V} y_{v} \cdot p_{v}} \\
& \times \prod_{i}\left[\int \frac{d^{D-d} k_{i}}{(2 \pi)^{D-d}} e^{-u_{i} k_{i}^{2}} e^{i \sum_{v=1}^{V} y_{v} \cdot \varepsilon_{v i} k_{i}}\right] \prod_{i}\left[\frac{1}{\prod_{\alpha} L_{\alpha}} \sum_{\substack{k_{i} \\
n_{i} \in \mathbb{Z} \\
\forall}} e^{-u_{i} \sum_{\alpha}\left(\omega_{\alpha}^{k_{i}}\right)^{2}} e^{i \sum_{v=1}^{V} \sum_{\alpha} z_{v}^{(\alpha)} \varepsilon_{v i} \omega_{\alpha}^{k_{i}}}\right] .
\end{aligned}
$$

Notice that the integral over $k_{i}$ is a Gaussian and we can complete the squares and compute the integral to get

$$
\int \frac{d^{D-d} k_{i}}{(2 \pi)^{D-d}} e^{-u_{i} k_{i}^{2}} e^{i \sum_{v=1}^{V} y_{v} \cdot \varepsilon_{v i} k_{i}}=\frac{e^{\left.-\frac{\left(\sum_{v=1}^{V} y_{v} \varepsilon_{v i}\right.}{4 u_{i}}\right)^{2}}}{\left(4 \pi u_{i}\right)^{\frac{D-d}{2}}},
$$

which, substituted back into $\tilde{\mathcal{I}}_{G}$, produces

$$
\begin{aligned}
\tilde{\mathcal{I}}_{G}= & C_{G}\left[\prod_{i} \int_{0}^{\infty} d u_{i}\right]\left[\prod_{v=1}^{V} \int d^{D-d} y_{v}\right]\left[\prod_{v=1}^{V} \prod_{\alpha} \int d z_{v}^{(\alpha)}\right] e^{-\sum_{i} u_{i} m_{i}^{2}} e^{-i \sum_{v=1}^{V} \sum_{\alpha} z_{v}^{(\alpha)} \omega_{\alpha}^{p_{v}}} e^{-i \sum_{v=1}^{V} y_{v} \cdot p_{v}} \\
& \times \prod_{i}\left[\frac{e^{-\frac{\left(\sum_{v=1}^{V} y_{v} \varepsilon_{v i}\right)^{2}}{4 u_{i}}}}{\left(4 \pi u_{i}\right)^{\frac{D-d}{2}}}\right] \prod_{i}\left[\frac{1}{\prod_{\alpha} L_{\alpha}} \sum_{\substack{k_{i} \in \mathbb{Z} \\
n_{i} \\
\forall<\alpha}} e^{-u_{i} \sum_{\alpha}\left(\omega_{\alpha}^{k_{i}}\right)^{2}+i \sum_{v=1}^{V} \sum_{\alpha} z_{v}^{(\alpha)} \varepsilon_{v i} \omega_{\alpha}^{k_{i}}}\right] .
\end{aligned}
$$

Now we make evident the global conservation required by the delta function. To do so we employ the change of variables,

$$
\begin{aligned}
& y_{v}=\bar{y}_{v}+y_{V}, \quad \forall v \neq V ; \\
& y_{V}=\bar{y}_{V} ; \\
& z_{v}^{(\alpha)}=\bar{z}_{v}^{(\alpha)}+z_{V}^{(\alpha)}, \quad \forall v \neq V ; \\
& z_{V}^{(\alpha)}=\bar{z}_{V}^{(\alpha)} .
\end{aligned}
$$

With this, the sum over all vertices produces 


$$
\sum_{v=1}^{V} y_{v} \varepsilon_{v i}=\sum_{v=1}^{V-1} \bar{y}_{v} \varepsilon_{v i}+\bar{y}_{V} \sum_{v=1}^{V} \varepsilon_{v i}=\sum_{v=1}^{V-1} \bar{y}_{v} \varepsilon_{v i}
$$

because $\sum_{v=1}^{V} \varepsilon_{v i}=0$ as a property of the incidence matrix (each internal line starts from one vertex +1 and ends at one vertex -1 , therefore the sum over all contributions is 0 ). Applying this change of variables,

$$
\begin{aligned}
& \tilde{\mathcal{I}}_{G}=C_{G}\left[\prod_{i} \int_{0}^{\infty} d u_{i}\right]\left[\prod_{v=1}^{V} \int d^{D-d} \bar{y}_{v}\right]\left[\prod_{v=1}^{V} \prod_{\alpha} \int d \bar{z}_{v}^{(\alpha)}\right] e^{-\sum_{i} u_{i} m_{i}^{2}} e^{-i \sum_{v=1}^{V-1} \sum_{\alpha} \bar{z}_{v}^{(\alpha)} \omega_{\alpha}^{p_{v}}} e^{-i \sum_{v=1}^{V-1} \bar{y}_{v} \cdot p_{v}}
\end{aligned}
$$

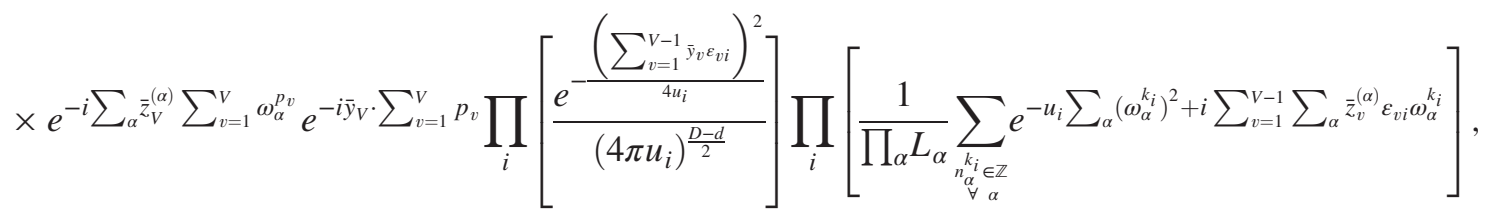

one can extract the global conservation (for the $v=V$ component),

$$
\int d^{D-d} \bar{y}_{V}\left[\prod_{\alpha} \int d \bar{z}_{V}^{(\alpha)}\right] e^{-i \sum_{\alpha} \bar{z}_{V}^{(\alpha)} \sum_{v=1}^{V} \omega_{\alpha}^{p_{v}}} e^{-i \bar{y}_{V} \cdot \sum_{v=1}^{V} p_{v}}=\delta^{D-d}\left(\sum_{v=1}^{V} p_{v}\right) \prod_{\alpha} \delta\left(\sum_{v=1}^{V} \omega_{\alpha}^{p_{v}}\right) .
$$

Due to this decomposition, it is usual to define a new amplitude without the overall conservation,

$$
\tilde{\mathcal{I}}_{G}=\delta^{D-d}\left(\sum_{v=1}^{V} p_{v}\right) \prod_{\alpha} \delta\left(\sum_{v=1}^{V} \omega_{\alpha}^{p_{v}}\right) \mathcal{I}_{G}
$$

such that,

$$
\begin{aligned}
& \mathcal{I}_{G}=C_{G}\left[\prod_{i} \int_{0}^{\infty} d u_{i}\right]\left[\prod_{v=1}^{V-1} \int d^{D-d} \bar{y}_{v}\right]\left[\prod_{v=1}^{V-1} \prod_{\alpha} \int d \bar{z}_{v}^{(\alpha)}\right] e^{-\sum_{i} u_{i} m_{i}^{2}} e^{-i \sum_{v=1}^{V-1} \sum_{\alpha} \bar{z}_{v}^{(\alpha)} \omega_{\alpha}^{p_{v}}} e^{-i \sum_{v=1}^{V-1} \bar{y}_{v} \cdot p_{v}} \\
& \times \prod_{i}\left[\frac{e^{\left.-\frac{\left(\sum_{v 1}^{V-1} \bar{y} v \varepsilon_{v i}\right.}{4 u_{i}}\right)^{2}}}{\left(4 \pi u_{i}\right)^{\frac{D-d}{2}}}\right] \prod_{i}\left[\frac{1}{\prod_{\alpha} L_{\alpha}} \sum_{\substack{k_{i} \in \mathbb{Z} \\
n_{\alpha} \in \alpha}} e^{-u_{i} \sum_{\alpha}\left(\omega_{\alpha}^{k_{i}}\right)^{2}+i \sum_{v=1}^{V-1} \sum_{\alpha} z_{v}^{(\alpha)} \varepsilon_{v i} \omega_{\alpha}^{k_{i}}}\right] .
\end{aligned}
$$

At this point we compute the gaussian integral over the parameter $\bar{y}_{v}$,

$$
\left[\prod_{v=1}^{V-1} \int d^{D-d} \bar{y}_{v}\right] e^{-i \sum_{v=1}^{V-1} \bar{y}_{v} \cdot p_{v}} e^{-\sum_{i} \frac{\left(\sum_{v=1}^{V-1} \bar{y}_{v} \varepsilon_{v i}\right)^{2}}{4 u_{i}}}=\frac{(4 \pi)^{\frac{(D-d)}{2}(V-1)}}{\left[\operatorname{det} d_{G}(u)\right]^{\frac{D-d}{2}}} e^{-\sum_{v_{1}, v_{2}=1}^{V-1} p_{v_{1}} p_{v_{2}}\left[d_{G}^{-1}(u)\right]_{v_{1}, v_{2}}} .
$$

Here, it was defined the symmetric $(V-1) \times(V-1)$ matrix $d_{G}(u)$ as

$$
\left[d_{G}(u)\right]_{v_{1}, v_{2}}=\sum_{i} \frac{\varepsilon_{v_{1} i} \varepsilon_{v_{2} i}}{u_{i}} .
$$

This produces, after a bit of organization,

$$
\mathcal{I}_{G}=C_{G}\left[\prod_{i} \int_{0}^{\infty} d u_{i}\right] \frac{e^{-\sum_{i} u_{i} m_{i}^{2}}}{(4 \pi)^{\frac{(D-d)}{2} L}} \frac{e^{-\frac{V(p)}{U}}}{U^{\frac{D-d}{2}}} \prod_{i}\left[\frac{1}{\prod_{\alpha} L_{\alpha}} \sum_{\substack{k_{i} \\ n_{i} \in \mathbb{Z} \\ \forall}}\right] \prod_{\alpha}\left\{\left[\prod_{v=1}^{V-1} \int d \bar{z}_{v}^{(\alpha)}\right] e^{-i \sum_{v=1}^{V-1} \bar{z}_{v}^{(\alpha)} \omega_{\alpha}^{p_{v}}} e^{-\sum_{i} u_{i}\left(\omega_{\alpha}^{k_{i}}\right)^{2}+i \sum_{v=1}^{V-1} \sum_{i} \bar{z}_{v}^{(\alpha)} \varepsilon_{v i} \omega_{\alpha}^{k_{i}}}\right\}
$$

or, introducing back the delta functions for the compactified dimensions, 


$$
\mathcal{I}_{G}=C_{G}\left[\prod_{i} \int_{0}^{\infty} d u_{i}\right] \frac{e^{-\sum_{i} u_{i} m_{i}^{2}}}{(4 \pi)^{\frac{(D-d)}{2}} L} \frac{e^{-\frac{V(p)}{U}}}{U^{\frac{D-d}{2}}} \prod_{i}\left[\frac{1}{\prod_{\alpha} L_{\alpha}} \sum_{\substack{k_{i} \\ n_{\alpha} \in \mathbb{Z} \\ \forall \alpha}} e^{-\sum_{\alpha} u_{i}\left(\omega_{\alpha}^{k_{i}}\right)^{2}}\right] \prod_{v=1}^{V-1} \prod_{\alpha}(2 \pi) \delta\left(\omega_{\alpha}^{p_{v}}-\sum_{i} \varepsilon_{v i} \omega_{\alpha}^{k_{i}}\right),
$$

where $L=I-V+1$ is the number of loops in the diagram (do not confuse with $L_{\alpha}$, the length of each compactified dimension), and the polynomials $U$ and $V$ are the Symanzik polynomials $[2,3]$, defined here as

$$
\begin{aligned}
\frac{V(q ; u)}{U(u)} & =\sum_{v_{1}, v_{2}=1}^{V-1} q_{v_{1}} q_{v_{2}}\left[d_{G}^{-1}(u)\right]_{v_{1}, v_{2}}, \\
U(u) & =\left(\prod_{i} u_{i}\right) \operatorname{det} d_{G}(u) .
\end{aligned}
$$

These polynomials have the remarkable property that they can be obtained directly from the graph $G$ by inspecting the 1-trees and 2-trees that can be formed by removing some internal lines. That is,

$$
\begin{aligned}
& U=\sum_{T} \prod_{i \notin T} u_{i} \\
& V=\sum_{K} Q_{K}^{2} \prod_{i \notin K} u_{i},
\end{aligned}
$$

meaning that the first polynomial $U$ is the sum over all 1-trees $T$ (connected graphs without loops) and we consider all lines $i$ that are not in the 1 -tree to be the removed lines. Also, the second polynomial $V$ is the sum over all 2-trees $K$ (two separated trees) where we take all parameters $u_{i}$ that do not belong to the 2-tree. $Q_{K}$ is the overall momenta that enters the 2-tree.

This ends the application of the usual procedure to the noncompactified dimensions. From now on we deal with the remaining $d$ dimensions. To proceed we employ the delta functions (in fact Kronecker deltas) to reduce the number of summations just to the loop summations and then treat the expression.

From this point forward $\prod_{v}$ refers to $\prod_{v=1}^{V-1}$ unless otherwise specified. As the sum is over the modes, one can extract a factor from the delta functions,

$$
\begin{aligned}
\mathcal{I}_{G}= & C_{G}\left[\prod_{i} \int_{0}^{\infty} d u_{i}\right] \frac{e^{-\sum_{i} u_{i} m_{i}^{2}}}{(4 \pi)^{\frac{(D-d)}{2} L}} \frac{e^{-\frac{V(p)}{U}}}{U^{\frac{D-d}{2}}} \\
& \times \prod_{\alpha}\left\{\frac{1}{L_{\alpha}^{I}} \sum_{\substack{k_{i} \in \mathbb{Z} \\
n_{\alpha} \\
\forall i}} e^{-\sum_{i} u_{i}\left(\omega_{\alpha}^{k_{i}}\right)^{2}}\left(L_{\alpha}\right)^{V-1}\right. \\
& \left.\times \prod_{v} \delta\left(n_{\alpha}^{p_{v}}-\sum_{i} \varepsilon_{v i} n_{\alpha}^{k_{i}}\right)\right\} .
\end{aligned}
$$

Notice that for each $\alpha$-th Matsubara mode we have $I$ summations (related to the internal lines) and $V-1$ relations between the frequencies (given by the Kronecker deltas), meaning an overall $L=I+1-V$ free frequencies to be summed up. This indicates that the notation could be changed to something like

$$
\begin{aligned}
T_{\alpha} & =\frac{1}{L_{\alpha}^{L}} \sum_{\substack{k_{i} \in \mathbb{Z} \\
n_{\alpha} \\
\forall i}} e^{-\sum_{i} u_{i}\left(\omega_{\alpha}^{k_{i}}\right)^{2}} \prod_{v} \delta\left(n_{\alpha}^{p_{v}}-\sum_{i} \varepsilon_{v i} n_{\alpha}^{k_{i}}\right) \\
& \equiv \frac{1}{L_{\alpha}^{L}} e^{-Z\left[\omega_{\alpha}^{p_{\nu}}\right]} \sum_{\substack{n_{\alpha}(\ell) \in \mathbb{Z} \\
\forall \\
\forall \in[1, L]}} e^{-Y\left[\omega_{\alpha}^{k_{\ell}}\right]},
\end{aligned}
$$

where the index $\ell$ runs over the $L$ independent loops. $Y\left[\omega_{\alpha}^{k_{\ell}}\right]$ is a bilinear form on the $L$ frequencies related to the loops, $Y$ is a $L \times L$ matrix whose coefficients depend on $u_{i}$, the external modes $n_{\alpha}^{p_{v}}$, and the incidence matrix. Also, $Z\left[\omega_{\alpha}^{p_{v}}\right]$ is another bilinear form that depends on the external modes. Let us now proceed to obtaining it using the delta functions. We need to rewrite in Eq. (19) the exponent

$$
\Delta_{\alpha}=-\sum_{i} u_{i}\left(\omega_{\alpha}^{k_{i}}\right)^{2}
$$

using the $V-1$ constraints given by the delta functions in Eq. (19),

$$
\omega_{\alpha}^{p_{v}}-\sum_{i} \varepsilon_{v i} \omega_{\alpha}^{k_{i}}=0
$$

We choose a prescription where the last lines will be eliminated. That is, we split the summation over all lines in Eq. (22) by a sum over the loops and the vertices $\left(\sum_{i}^{I}=\sum_{\ell}^{L}+\sum_{v}^{V-1}\right.$, as $\left.I=L+V-1\right)$. With this, we can use the constraints to define a linear system,

$$
\omega_{\alpha}^{p_{v}}-\sum_{\ell} \varepsilon_{v \ell} \omega_{\alpha}^{k_{\ell}}=\sum_{v_{0}=1}^{V-1} \varepsilon_{v, L+v_{0}} \omega_{\alpha}^{k_{L+v_{0}}},
$$

that determines the frequencies to be eliminated $\left(\omega_{\alpha}^{k_{L+\nu_{0}}}\right)$ as a function of the $L$ frequencies to be kept $\left(\omega_{\alpha}^{k_{\ell}}\right)$. In matrix notation, this means trivially that

$$
Q=\bar{\epsilon} \bar{W} \Rightarrow \bar{W}=\bar{\epsilon}^{-1} Q,
$$

with 


$$
\begin{gathered}
(Q)_{v}=\omega_{\alpha}^{p_{v}}-\sum_{\ell} \varepsilon_{v \ell} \omega_{\alpha}^{k_{\ell}}, \\
(\bar{\epsilon})_{v, v_{0}}=\varepsilon_{v, L+v_{0}}, \\
(\bar{W})_{v_{0}}=\omega_{\alpha}^{k_{L+v_{0}}} .
\end{gathered}
$$

Of course, for this to work, we need the $(V-1) \times(V-1)$ matrix $\bar{\epsilon}$ to be invertible. This is not guaranteed for all choices of labeling of lines and vertices when building the incidence matrix $\varepsilon_{v, i}$. But we can always reorganize the labels to guarantee a choice where $\bar{\epsilon}$ is invertible. There are a few remarks here. First, here we always choose to eliminate the last internal lines, this choice does not affect the results, and is just a matter of aesthetics (we want to keep the same labels for the number of loops, so the $L$ frequencies related to the $L$ loops are exactly the first $L$ internal lines). Second, different choices of $\bar{\epsilon}$ will not affect the appearance of the Symanzik polynomials $U$ and $V$, as one should expect for consistency. However, the matrix $Y$ will depend on this choice. Anyway, one can show that through a suitable rearrangement of the modes $\left(n_{\alpha}^{k_{\ell}}\right)$ one can relate a matrix $Y$ obtained by some $\bar{\epsilon}$ to another matrix $Y$ obtained by another choice of $\bar{\epsilon}$. Therefore, we do not lose generality by specifying some prescriptions to obtain $\bar{\epsilon}$.

If we substitute Eq. (24a) back into Eq. (21),

$$
\Delta_{\alpha}=-\sum_{\ell} u_{\ell}\left(\omega_{\alpha}^{k_{\ell}}\right)^{2}-\sum_{v_{0}=1}^{V-1} u_{L+v_{0}}\left(\omega_{\alpha}^{k_{L+v_{0}}}\right)^{2}=-\sum_{\ell} u_{\ell}\left(\omega_{\alpha}^{k_{\ell}}\right)^{2}-\sum_{v_{0}, v_{1}, v_{2}=1}^{V-1} u_{L+v_{0}}\left[\left(\bar{\epsilon}^{-1}\right)_{v_{0}, v_{1}} Q_{v_{1}}\right]\left[\left(\bar{\epsilon}^{-1}\right)_{v_{0}, v_{2}} Q_{v_{2}}\right],
$$

meaning that $T_{\alpha}$, Eq. (20), becomes

$$
T_{\alpha}=\frac{1}{L_{\alpha}^{L}} \sum_{\substack{k_{\ell} \in \mathbb{Z} \\ n_{\alpha} \in \mathcal{Z}}} e^{-\sum_{e^{\prime}} u_{\ell}\left(\omega_{\alpha}^{k}\right)^{2}-\sum_{v_{0}, v_{1}, v_{2}=1}^{V-1} u_{L+v_{0}}\left[\left(\bar{\epsilon}^{-1}\right)_{v_{0}, v_{1}} Q_{v_{1}}\right]\left[\left(\bar{\epsilon}^{-1}\right)_{v_{0}, v_{2}} Q_{v_{2}}\right] .}
$$

Notice that the Kronecker delta functions eliminated $V-1$ of the summations. To proceed we need to reorganize the expression to make evident the component that depends on $\omega_{\alpha}^{k_{\ell}}$ and the component that is independent of it. To do so we apply the expression of $Q_{v}$, Eq. (24b),

$$
\Delta_{\alpha}=-\sum_{\ell} u_{\ell}\left(\omega_{\alpha}^{k_{\ell}}\right)^{2}-\sum_{v_{0}, v_{1}, v_{2}=1}^{V-1} u_{L+v_{0}}\left[\left(\bar{\epsilon}^{-1}\right)_{v_{0}, v_{1}}\left(\omega_{\alpha}^{p_{v_{1}}}-\sum_{\ell_{1}} \varepsilon_{v_{1} \ell_{1}} \omega_{\alpha}^{k_{\ell_{1}}}\right)\right]\left[\left(\bar{\epsilon}^{-1}\right)_{v_{0}, v_{2}}\left(\omega_{\alpha}^{p_{v_{2}}}-\sum_{\ell_{2}} \varepsilon_{v_{2} \ell_{2}} \omega_{\alpha}^{k_{\ell_{2}}}\right)\right],
$$

and open all its terms explicitly,

$$
\begin{aligned}
\Delta_{\alpha}= & -\sum_{\ell_{1}, \ell_{2}} \omega_{\alpha}^{k_{\ell_{1}}}\left[u_{\ell_{1}} \delta_{\ell_{1}, \ell_{2}}+\sum_{v_{0}, v_{1}, v_{2}=1}^{V-1} u_{L+v_{0}}\left(\bar{\epsilon}^{-1}\right)_{v_{0}, v_{1}} \varepsilon_{v_{1} \ell_{1}}\left(\bar{\epsilon}^{-1}\right)_{v_{0}, v_{2}} \varepsilon_{v_{2} \ell_{2}}\right] \omega_{\alpha}^{k_{\ell_{2}}} \\
& +\sum_{\ell} \omega_{\alpha}^{k_{\ell}} \sum_{v_{0}, v_{1}, v_{2}=1}^{V-1} u_{L+v_{0}}\left[\left(\bar{\epsilon}^{-1}\right)_{v_{0}, v_{1}} \omega_{\alpha}^{p_{v_{1}}}\left(\bar{\epsilon}^{-1}\right)_{v_{0}, v_{2}} \varepsilon_{v_{2} \ell_{2}}+\left(\bar{\epsilon}^{-1}\right)_{v_{0}, v_{1}} \varepsilon_{v_{1} \ell}\left(\bar{\epsilon}^{-1}\right)_{v_{0}, v_{2}} \omega_{\alpha}^{p_{v_{2}}}\right] \\
& -\sum_{v_{0}, v_{1}, v_{2}=1}^{V-1} u_{L+v_{0}}\left[\left(\bar{\epsilon}^{-1}\right)_{v_{0}, v_{1}} \omega_{\alpha}^{p_{v_{1}}}\right]\left[\left(\bar{\epsilon}^{-1}\right)_{v_{0}, v_{2}} \omega_{\alpha}^{p_{v_{2}}}\right] .
\end{aligned}
$$

To simplify the notation we define $W_{\alpha}, M, B_{\alpha}$, and $F_{\alpha}$ as

$$
\begin{aligned}
\left(W_{\alpha}\right)_{\ell} & =\omega_{\alpha}^{k_{\ell}}, \\
(M)_{\ell_{1}, \ell_{2}} & =u_{\ell_{1}} \delta_{\ell_{1}, \ell_{2}}+\sum_{v_{0}, v_{1}, v_{2}} u_{L+v_{0}}\left[\left(\bar{\epsilon}^{-1}\right)_{v_{0}, v_{1}} \varepsilon_{v_{1}, \ell_{1}}\right]\left[\left(\bar{\epsilon}^{-1}\right)_{v_{0}, v_{2}} \varepsilon_{v_{2}, \ell_{2}}\right], \\
\left(B_{\alpha}\right)_{\ell} & =\sum_{v_{0}, v_{1}, v_{2}} u_{L+v_{0}}\left[\left(\bar{\epsilon}^{-1}\right)_{v_{0}, v_{1}} \varepsilon_{v_{1}, \ell}\right]\left[\left(\bar{\epsilon}^{-1}\right)_{v_{0}, v_{2}} \omega_{\alpha}^{p_{v_{2}}}\right], \\
F_{\alpha} & =\sum_{v_{0}, v_{1}, v_{2}} u_{L+v_{0}}\left[\left(\bar{\epsilon}^{-1}\right)_{v_{0}, v_{1}} \omega_{\alpha}^{p_{v_{1}}}\right]\left[\left(\bar{\epsilon}^{-1}\right)_{v_{0}, v_{2}} \omega_{\alpha}^{p_{v_{2}}}\right] .
\end{aligned}
$$


Here, $M$ is a $L \times L$ matrix. With this notation we can write $\Delta_{\alpha}$ as

$$
\Delta_{\alpha}=-W_{\alpha}^{t} M W_{\alpha}+W_{\alpha}^{t} B_{\alpha}+B_{\alpha}^{t} W_{\alpha}-F_{\alpha},
$$

and complete the squares to obtain

$$
\begin{aligned}
\Delta_{\alpha}= & -\left(W_{\alpha}-M^{-1} B_{\alpha}\right)^{t} M\left(W_{\alpha}-M^{-1} B_{\alpha}\right) \\
& +\left(-F_{\alpha}+B_{\alpha}^{t} M^{-1} B_{\alpha}\right) .
\end{aligned}
$$

For convenience, we can also define a tilde notation to indicate that the factor $2 \pi / L_{\alpha}$ is extracted, that is

$$
\left(\widetilde{W}_{\alpha}\right)_{\ell}=n_{\alpha}^{k_{\ell}}
$$

$$
\left(\tilde{B}_{\alpha}\right)_{\ell}=\sum_{v_{0}, v_{1}, v_{2}} u_{L+v_{0}}\left[\left(\bar{\epsilon}^{-1}\right)_{v_{0}, v_{1}} \varepsilon_{v_{1}, \ell}\right]\left[\left(\bar{\epsilon}^{-1}\right)_{v_{0}, v_{2}} n_{\alpha}^{p_{v_{2}}}\right] .
$$

At this point $T_{\alpha}$, Eq. (25), is written as

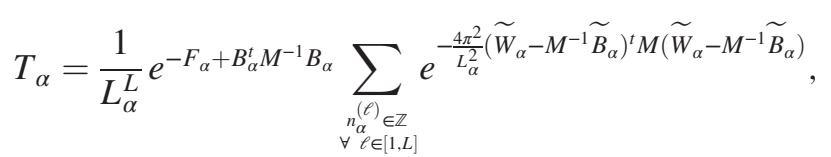

and we can get back to the original expression, Eq. (19), and rewrite the amplitude related to some graph $G$ as

$$
\mathcal{I}_{G}=C_{G}\left[\prod_{i} \int_{0}^{\infty} d u_{i}\right] \frac{e^{-\sum_{i} u_{i} m_{i}^{2}}}{(4 \pi)^{\frac{(D-d)}{2} L}} \frac{e^{-\frac{V(p)}{U}}}{U^{\frac{D-d}{2}}} \prod_{\alpha}\left\{\frac{1}{L_{\alpha}^{L}} e^{-F_{\alpha}+B_{\alpha}^{t} M^{-1} B_{\alpha}} \sum_{\substack{n_{\alpha}^{(\ell)} \in \mathbb{Z} \\ \forall \ell \in[1, L]}} e^{-\frac{4 \pi^{2}}{L_{\alpha}^{2}}\left(\widetilde{W}_{\alpha}-M^{-1} \widetilde{B}_{\alpha}\right)^{t} M\left(\widetilde{W}_{\alpha}-M^{-1} \widetilde{B}_{\alpha}\right)}\right\} .
$$

With the previous procedure (integrate over the momenta and then integrate over the parameters related to the delta function) we found the standard definition of the Symanzik polynomials [see Eq. (17)] in terms of the incidence matrix. Using the alternative procedure (apply first the delta function and then reduce the number of integrals) the Symanzik polynomials can be expressed $a^{2}$

$$
\begin{aligned}
U(u) & =\operatorname{det} M, \\
\frac{V\left(\omega_{\alpha}^{p}, u\right)}{U(u)} & =F_{\alpha}-B_{\alpha}^{t} M^{-1} B_{\alpha},
\end{aligned}
$$

with $M, B$, and $F$ defined as in Eq. (28). Therefore, one can simplify the representation to

$$
\begin{aligned}
\mathcal{I}_{G}= & C_{G}\left[\prod_{i} \int_{0}^{\infty} d u_{i}\right] \frac{e^{-\sum_{i} u_{i} m_{i}^{2}}}{(4 \pi)^{\frac{(D-d)}{2} L}} \frac{e^{-\frac{V(P)}{U}}}{U^{\frac{D-d}{2}}} \\
& \times \prod_{\alpha}\left\{\frac{1}{L_{\alpha}^{L}} \sum_{\substack{n_{\alpha}^{(\ell)} \in \mathbb{Z} \\
\forall \in \in[1, L]}} e^{\left.-\frac{4 \pi^{2}}{L_{\alpha}^{2}} \widetilde{W}_{\alpha}-M^{-1} \widetilde{B}_{\alpha}\right)^{t} M\left(\widetilde{W}_{\alpha}-M^{-1} \widetilde{B}_{\alpha}\right)}\right\} .
\end{aligned}
$$

Remember that $P_{v}=\left(p_{v}, \omega_{\alpha}^{p_{v}}\right)$ is the full external momenta, meaning that $V(P)=V(p)+\sum_{\alpha} V\left(\omega_{\alpha}^{p}\right)$.

From this point forward we will consider two different scenarios: the small-box regime that approaches dimensional reduction $\left(\Lambda L_{\alpha} \ll 1\right.$, where $\Lambda$ is some mass scale)

\footnotetext{
${ }^{2}$ One can check that both representations are equivalent by repeating the procedure for the noncompact dimensions. This is discussed in Ref. [78].
}

and the large-box regime that approaches the bulk where no compactification occurs $\left(\Lambda L_{\alpha} \gg 1\right)$. Both of them are equivalent analytically, in the sense that it is possible to transport from one to another, but each of the representations is more suitable for a different length scale. At first, we show the behavior when the compactification lengths are small (near a dimensional reduction); in this scenario the expression is exactly the one from Eq. (34) but requires some treatment, which is done in Sec. III. Then, in Sec. IV, we show the behavior when the compactification lengths are large (near the bulk); for this we employ a Jacobi theta identification that modifies the summation in Eq. (34). The difference between both procedures is that, although both are indeed valid for all compactified sizes, each one converges faster on the specified limit. That said, although it can be done in principle it is not indicated to employ the representation from Sec. III to study the bulk limit, nor the representation from Sec. IV to study the dimensional reduction limit.

\section{NEAR DIMENSIONAL REDUCTION REPRESENTATION}

As far as the author knows, although the topic of dimensional reduction is well known in the subject of finite temperature field theory (a dimensional reduction occurs in the very high-temperature limit) the only attempt to understand the behavior of a Feynman amplitude with many periodically compactified spaces near a dimensional reduction comes from $[76,77]$. The present context, where we produce a parametric representation for a scalar field theory in a periodically compactified space, allows a clear and easier evaluation of the dimensional reduction. 
At first, let us make clear that we consider the dimensional reduction in the sense of Fisher [79]. That is, one does not say that all length parameters are zero $\left(L_{\alpha}=0\right)$, but rather that the length is small enough so that its contribution can be mostly ignored. So we can take the limit where $L_{\alpha} \rightarrow 0$ and keep track only of the dominant contribution. We can be a bit more careful and say, instead, that we first take a small box, defined in such a way that the length of each side satisfies $\Lambda L_{\alpha} \ll 1$, where $\Lambda$ is some momentum scale, and the dimensional reduction is the dominant contribution in the small-box regime. Let us take the amplitude $I_{G}$ as in Eq. (34) and put $L_{\alpha}$ in evidence,

$$
\mathcal{I}_{G}=C_{G}\left[\prod_{i} \int_{0}^{\infty} d u_{i}\right] \frac{e^{-\sum_{i} u_{i} m_{i}^{2}}}{(4 \pi)^{\frac{(D-d)}{2}} L} \frac{e^{-\frac{V(p)}{U}}}{U^{\frac{D-d}{2}}} \prod_{\alpha}\left\{\sum_{\substack{(\alpha) \in \mathbb{Z} \\ \forall \in \in[1, L]}} \frac{e^{-\frac{4 \pi^{2}}{L_{\alpha}^{2}} G_{\alpha}}}{L_{\alpha}^{L}}\right\} .
$$

The function $G_{\alpha}$ is independent of $L_{\alpha}$, it depends on the Schwinger parameters $u_{i}$ and the external modes $n_{\alpha}^{p_{v}}$ as

$G_{\alpha}=\widetilde{F}_{\alpha}-\widetilde{B}_{\alpha}^{t} M^{-1} \widetilde{B}_{\alpha}+\left(\widetilde{W}_{\alpha}-M^{-1} \widetilde{B}_{\alpha}\right)^{t} M\left(\widetilde{W}_{\alpha}-M^{-1} \widetilde{B}_{\alpha}\right)$.

Recall the definitions of $F_{\alpha}, B_{\alpha}$, and $M$ in Eq. (28). By dimensional analysis we can get that the Schwinger parameters $u_{i}$ have the dimension of inverse squared mass, $\left[u_{i}\right]=\Lambda^{-2}$, and by inspection of $G_{\alpha}$ we obtain that it also behaves as $\left[G_{\alpha}\right]=\Lambda^{-2}$. That is, the exponent with dependence on $L_{\alpha}$ could be written as $-4 \pi^{2} /\left(\Lambda L_{\alpha}\right)^{2}$ times some dimensionless component. The small-box approximation $\Lambda L_{\alpha} \ll 1$ means here an exponential suppression. The dominant contribution from the whole series in the internal modes $n_{\alpha}^{(\ell)}$ is, therefore, the set of modes that minimize the function $G_{\alpha}$, that is

$\mathcal{I}_{G} \sim C_{G}\left[\prod_{i} \int_{0}^{\infty} d u_{i}\right] \frac{e^{-\sum_{i} u_{i} m_{i}^{2}}}{(4 \pi)^{\frac{(D-d)}{2} L}} \frac{e^{-\frac{V(p)}{U}}}{U^{\frac{D-d}{2}}} \prod_{\alpha}\left[\frac{e^{-\frac{4 \pi^{2}}{L_{\alpha}^{2}} \min G_{\alpha}}}{L_{\alpha}^{L}}\right]$.

The minimization with respect to the internal modes means that one chooses a set $\widetilde{W}_{\alpha}$ such that $G_{\alpha}$ has its minimum value. If $M^{-1} \widetilde{B}_{\alpha}=0$ this is trivially $\widetilde{W}_{\alpha}=0$. The component $L_{\alpha}^{L}$ can be easily absorbed by the definition of the coupling constant (that is inside the constant $C_{G}$ ), but $G_{\alpha}$ is not easily absorbed. This component, when $\widetilde{W}_{\alpha} \neq 0$ for at least one $\alpha$, introduces a new contribution to the Symanzik polynomials coming from a dynamic mode $(n \neq 0)$. That is, in the limit of dimensional reduction one does not simply get the same amplitude in reduced dimensions, but there can be some surviving information from the original dimensions. This becomes more explicit when dealing with examples. In Sec. V we consider a scalar $\phi^{3}$ model where the one-loop contribution at $D=4, d=2$ (one compactification related to the inverse temperature $\beta$ and the other to a spatial compactification $L_{1}$ ) is given by Eq. (65),

$$
\left.\mathcal{I}_{1}\right|_{\text {dim.red. }} \sim \frac{C_{1}}{2 \beta L_{1}} \frac{\ln \left(q^{2}+\omega^{2}+3 m^{2}\right)}{q^{2}+\omega^{2}+2 m^{2}} .
$$

The contribution from the external modes (given here by $\omega^{2}$ ) acts as a shift in the mass. This can be understood as a generalization of the notion of a thermal shift in the mass of a particle $[80,81]$. We see that all compactified dimensions can contribute to this effective mass and that it naturally occurs if one goes into the small-box limit of the system.

Sometimes, one can also assume another perspective: a completely static mode approach. In this perspective all modes, internal and external, are null, and this makes $G_{\alpha}=0$ by producing simply

$\left.\mathcal{I}_{G}\right|_{\left\{n_{\alpha}^{k_{\ell}}, n_{\alpha}^{p_{v}}\right\}=0}=\frac{C_{G}}{\prod_{\alpha} L_{\alpha}^{L}}\left[\prod_{i} \int_{0}^{\infty} d u_{i}\right] \frac{e^{-\sum_{i} u_{i} m_{i}^{2}}}{(4 \pi)^{\frac{(D-d)}{2} L}} \frac{e^{-\frac{V(p)}{U}}}{U^{\frac{D-d}{2}}}$.

Notice we can absorb the remaining factor related with the size in the constant $C_{G}$ associated with the vertices. This is exactly the usual parametric representation of a Feynman diagram but only considering the $D-d$ noncompactified and large dimensions. In this perspective one can easily establish a prescription to identify $D \rightarrow D-d$ and then divide by a factor $\prod_{\alpha} L_{\alpha}^{L}$ to obtain the dimensionally reduced amplitude. Notice that this approach means a fine-tuned dimensional reduction such that no "effective mass" appears due to the external modes of the compactified dimensions.

Sometimes it might look like both procedures are equivalent. The difference between them becomes more evident if we consider a scenario where the graph $G$ is a subdiagram to be evaluated in the ultraviolet regime; in this scenario the "external" momenta for the subdiagram are internal momenta for the full diagram and can be arbitrarily large, so there is no justification to use a zero-mode approximation for the "external" frequencies of the subdiagram. This simple remark can be responsible to produce large differences in behavior.

The remaining terms, subdominant contributions, to the amplitude in the small length regime are obtained in a straightforward way. Just as the dominant contribution is the minimum with respect to the internal modes $\left(G_{\alpha}\right)$, the next leading order is a small deviation around this minimum. To make notation clear we apply a shift in the internal modes, that is

$$
n_{\alpha}^{(\ell)} \rightarrow n_{\alpha}^{(\ell)}+j_{\alpha}^{\ell},
$$


where $j_{\alpha}^{\ell} \in \mathbb{Z}$. This is chosen such that the minimum now lies on $n_{\alpha}^{(\ell)}=0$. Let us write this shift as

$$
\widetilde{W}_{\alpha} \rightarrow \widetilde{W}_{\alpha}-\widetilde{Y}_{\alpha}=\widetilde{W}_{\alpha}+M^{-1} \widetilde{B}_{\alpha}-\widetilde{Z}_{\alpha},
$$

so that $G_{\alpha}$ can be rewritten as

$$
G_{\alpha}=\widetilde{F}_{\alpha}-\widetilde{B}_{\alpha}^{t} M^{-1} \widetilde{B}_{\alpha}+\left(\widetilde{W}_{\alpha}-\widetilde{Z}_{\alpha}\right)^{t} M\left(\widetilde{W}_{\alpha}-\widetilde{Z}_{\alpha}\right) .
$$

We must choose a vector $\widetilde{Y}_{\alpha} \in \mathbb{Z}^{L}$ such that the components of the resultant vector $\widetilde{Z}_{\alpha}$ all lie in the interval $\left(\widetilde{Z}_{\alpha}\right)_{\ell} \in\left[-\frac{1}{2}, \frac{1}{2}\right]$. This guarantees that the minimum value of
$G_{\alpha}$ occurs at $\widetilde{W}_{\alpha}=0$. The simple choice is that $\widetilde{Y}_{\alpha}$ is the nearest integer to the real value $M^{-1} \widetilde{B}_{\alpha}$, which we denote by $\left\lfloor M^{-1} \widetilde{B}_{\alpha}\right\rceil$. Therefore, the vector $\widetilde{Z}_{\alpha}$ is just

$$
\widetilde{Z}_{\alpha}=M^{-1} \widetilde{B}_{\alpha}-\left\lfloor M^{-1} \widetilde{B}_{\alpha}\right\rceil .
$$

With this simple change of notation the order of dominance is easier to write. The dominant contribution, as already stated, occurs at $\sum_{\ell}\left|n_{\alpha}^{(\ell)}\right|=0$, meaning that $n_{\alpha}^{(\ell)}=0, \quad \forall \ell$. The first correction occurs at $\sum_{\ell}\left|n_{\alpha}^{(\ell)}\right|=1$ and so on. This produces the simple expression,

$$
\begin{aligned}
& \mathcal{I}_{G}=C_{G}\left[\prod_{i} \int_{0}^{\infty} d u_{i}\right] \frac{e^{-\sum_{i} u_{i} m_{i}^{2}}}{(4 \pi)^{\frac{(D-d)}{2} L}} \frac{e^{-\frac{V(P)}{U}}}{U^{\frac{D-d}{2}}} \prod_{\alpha}\left\{\frac{1}{L_{\alpha}^{L}} e^{-Z_{\alpha}^{t} M Z_{\alpha}}\right\}
\end{aligned}
$$

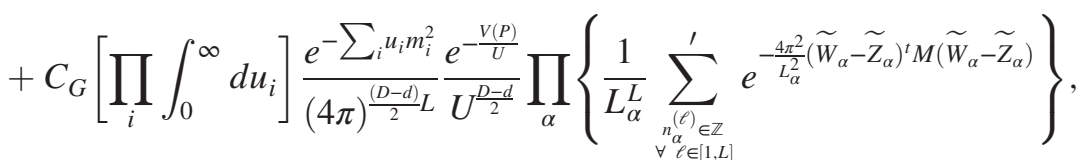

where $\sum^{\prime}$ denotes that the zeroth mode is already removed, and we put $G_{\alpha}$ explicitly such that the contribution for the second Symanzik polynomial $V$ depends on the full $D$-dimensional momentum $P$ instead of the remaining continuum $(D-d)$-dimensional momentum $p$.

In the next section, we deal with the scenario near the bulk (large compactification length) and rewrite the parametrization using a new distribution of sectors that change the $u$ parameters to $t$ parameters. Near the bulk, this reparametrization has the advantage of letting us see the appearance of the modified Bessel function of the second kind, characteristic of periodically compactified problems. However, when discussing the scenario near dimensional reduction, this new parametrization does not contribute a lot, so we leave the explanation of it for the next section. For completeness, however, we show below how the amplitude looks after this reparametrization:

$$
\begin{aligned}
\mathcal{I}_{G}= & \frac{1}{\left(\prod_{\alpha} L_{\alpha}\right)^{L}} C_{G}\left[\prod_{i=1}^{I-1} \int_{0}^{1} d t_{i} t_{i}^{I-1-i}\right] \frac{\Gamma\left(I-\frac{(D-d) L}{2}\right)}{(4 \pi)^{\frac{(D-d)}{2} L} \bar{U}^{\frac{(D-d)}{2}}} \frac{1}{\left[\mathcal{M}^{2}\left(t_{i}, m_{i}\right)+\frac{\bar{V}(P ; t)}{\bar{U}(t)}+\bar{Z}_{\alpha}^{t} \bar{M} \bar{Z}_{\alpha}\right]^{I-\frac{(D-d) L}{2}}} \\
& +\frac{1}{\left(\prod_{\alpha} L_{\alpha}\right)^{L}} C_{G}\left[\prod_{i=1}^{I-1} \int_{0}^{1} d t_{i} t_{i}^{I-1-i}\right] \frac{\Gamma\left(I-\frac{(D-d) L}{2}\right)}{(4 \pi)^{\frac{(D-d)}{2} L} \bar{U}^{(D-d)}} \times \sum_{\substack{n_{\alpha}^{(\ell)} \in \mathbb{Z} \\
\forall \ell \in, \forall}}^{1} \frac{1}{\left[\mathcal{M}^{2}\left(t_{i}, m_{i}\right)+\frac{\bar{V}(P ; t)}{\bar{U}(t)}+\left(\bar{W}_{\alpha}-\bar{Z}_{\alpha}\right)^{t} \bar{M}\left(\bar{W}_{\alpha}-\bar{Z}_{\alpha}\right)\right]^{I-\frac{(D-d) L}{2}}}
\end{aligned}
$$

The remaining sum is a zetalike summation, in the scenario of very small compactification lengths, it can be truncated at low terms (as discussed before, each new contribution is subdominant).

\section{NEAR BULK REPRESENTATION}

In the previous section we considered the system in a small box, which can deal with the scenario near the dimensional reduction. In this section we consider the large-box scenario, that shall reproduce the expression near the bulk where no compactification exists. We can naively think of the near bulk scenario as taking the limit $L_{\alpha} \rightarrow \infty$.
In a more careful treatment this shall be understood, in fact, as $\Lambda L_{\alpha} \gg 1$, where $\Lambda$ is some mass scale. In the original expression, Eq. (36), this produces a dependence like $e^{-1 /\left(\Lambda L_{\alpha}\right)^{2}}$, meaning that all exponential weights are equally large, and this slows down the convergence of the series. We can achieve a more suitable expression by employing the Jacobi theta identity,

$$
\begin{aligned}
& \sum_{\mathbf{a} \in \mathbb{Z}^{n}} e^{-\pi t Y[\mathbf{a}+\mathbf{g}]} e^{2 \pi i \mathbf{h} \cdot \mathbf{a}} \cdot \\
& \quad=e^{-2 \pi i \mathbf{h} \cdot \mathbf{g}} t^{-\frac{n}{2}}|Y|^{-\frac{1}{2}} \sum_{\mathbf{a} \in \mathbb{Z}^{n}} e^{-\frac{\pi}{t} Y^{-1}[\mathbf{a}-\mathbf{h}]} e^{2 \pi i \mathbf{a} \cdot \mathbf{g}},
\end{aligned}
$$


with $t=4 \pi / L_{\alpha}^{2}, n=L$, the matrix $Y=M,(\mathbf{g})_{n}=-\left(M^{-1} \widetilde{B}_{\alpha}\right)_{\ell}$, and $(\mathbf{a})_{n}=\left(\widetilde{W}_{\alpha}\right)_{\ell}=n_{\alpha}^{k_{\ell}}$, so that we obtain

$$
\mathcal{I}_{G}=C_{G}\left[\prod_{i} \int_{0}^{\infty} d u_{i}\right] \frac{e^{-\sum_{i} u_{i} m_{i}^{2}}}{(4 \pi)^{\frac{(D-d)}{2} L}} \frac{e^{-\frac{V(P)}{U}}}{U^{\frac{D-d}{2}}} \prod_{\alpha}\left\{\frac{1}{(4 \pi)^{\frac{L}{2}}[\operatorname{det} M]^{\frac{1}{2}}} \sum_{\substack{n_{\alpha}^{(\ell)} \in \mathbb{Z} \\ \forall \ell \in[1, L]}} e^{-\frac{L_{\alpha}^{2} \widetilde{W}_{\alpha}^{t}}{4} M^{-1} \widetilde{\widetilde{W}}_{\alpha}} e^{2 \pi i \widetilde{W}_{\alpha}^{t} M^{-1} \widetilde{B}_{\alpha}}\right\}
$$

Notice that now we have a dependence like $e^{-\left(\Lambda L_{\alpha}\right)^{2}}$, meaning that each new mode introduces a bigger suppression and the sum over the modes is expected to converge fast in the chosen regime (large box / near bulk). Recall at this point that $P$ is the $D$-dimensional external momentum composed by the continuum $(D-d)$-dimensional momentum $p$ and the $d$ discrete frequencies $\omega_{\alpha}^{p}$.

We can rewrite the amplitude of a graph $G$ with $d$ compactified dimensions recalling that $U=\operatorname{det} M$, writing explicitly the modes and defining the matrix $A=M^{-1} \operatorname{det} M$, that is

$$
\mathcal{I}_{G}=C_{G}\left[\prod_{i} \int_{0}^{\infty} d u_{i}\right] \frac{e^{-\sum_{i} u_{i} m_{i}^{2}}}{(4 \pi)^{\frac{D}{2} L}} \frac{e^{-\frac{V(P(P) u)}{U(u)}}}{U(u)^{\frac{D}{2}}} \times \sum_{\substack{u(\ell) \\ n_{\alpha} \in \mathbb{Z} \\ \forall \ell, \forall \alpha}} e^{-\sum_{\alpha} \frac{L_{\alpha}^{2}}{4 U(u)} \sum_{\ell_{1}, \ell_{2}} n_{\alpha}^{k_{\ell_{1}}} n_{\alpha}^{k_{\ell}} A_{\ell_{1}, \ell_{2}}(u)} e^{\frac{2 \pi i}{U(u)} \sum_{\alpha, \ell_{1}, \ell_{2}} n_{\alpha}^{k_{\ell}} A_{\ell_{1}, \ell_{2}}(u)\left(\widetilde{B}_{\alpha}(u)\right)_{\ell_{2}}}
$$

or, in compact notation,

$$
\mathcal{I}_{G}=C_{G}\left[\prod_{i} \int_{0}^{\infty} d u_{i}\right] \frac{e^{-\sum_{i} u_{i} m_{i}^{2}}}{(4 \pi)^{\frac{D}{2} L}} \frac{e^{-\frac{V(P, u)}{U(u)}}}{U(u)^{\frac{D}{2}}} \sum_{\substack{n_{\alpha}(u) \in \mathbb{Z} \\ \forall \\ \forall, \forall \alpha}} e^{-\sum_{\alpha} \frac{L_{\alpha}^{2}}{4 U(u)} \mathbf{n}_{\alpha}^{t} A(u) \mathbf{n}_{\alpha}} e^{\frac{2 \pi i}{U(u)} \sum_{\alpha} \mathbf{n}_{\alpha}^{t} A(u) \widetilde{B}_{\alpha}(u)} .
$$

Here $\mathbf{n}_{\alpha}$ is vector with components $\left(\mathbf{n}_{\alpha}\right)_{\ell}=n_{\alpha}^{k_{\ell}}$.

Based on all we have done so far, the prescription one must follow is:

(1) From the graph extract the 1-trees and produce $U(u)$ as usual, see Eq. (18);

(2) From the graph extract the 2-trees and produce $V(u)$ as usual, see Eq. (18);

(3) From the incidence matrix $\varepsilon_{v, i}$ extract a $(V-1) \times$ $(V-1)$ matrix $\bar{\epsilon}$ that is invertible. By convention, take the last internal lines;

(4) Using $\bar{\epsilon}, \varepsilon_{v, i}$, and $\omega_{\alpha}^{p_{v}}$ compute the $M$, Eq. (28b), and $\widetilde{B}$, Eq. (32). The matrix $A$ is defined as $A=M^{-1} \operatorname{det} M$.

Unfortunately, up to this point, we do not have a prescription that allows us to recover the matrix $A$ or the vector $\tilde{B}$ directly from the topology of the diagram, like $U$ and $V$ that are extracted by drawing the 1-trees and 2-trees related to the graph [see Eq. (18)].

The Symanzyk polynomials $U$ and $V$ are solely related to the graph, however, we also need $A$ and $B$, which are determined from our prescription of $\bar{\epsilon}$. One might inquire whether they are different depending on the choice of prescription and indeed they are. Unlike $U$ and $V$, which do not feel our choice of $\bar{\epsilon}$, the factors $A$ and $B$ depend on it. However, we can transform the summation modes in such a way to transform one representation to the other, meaning that they are all equivalent.

One can easily notice from Eq. (50) that the zeroth mode now represents the bulk scenario (if we take $L_{\alpha} \rightarrow \infty$ we get an exponential suppression, so the only survivor is $\left.n_{\alpha}^{(\ell)}=0\right)$. This will produce the standard representation,

$$
\left.\mathcal{I}_{G}\right|_{\text {bulk }} \sim C_{G}\left[\prod_{i} \int_{0}^{\infty} d u_{i}\right] \frac{e^{-\sum_{i} u_{i} m_{i}^{2}}}{(4 \pi)^{\frac{D}{2} L}} \frac{e^{-\frac{V(P, u)}{U(u)}}}{U(u)^{\frac{D}{2}}}
$$

as should be expected. This shows the consistency of the representation, which recovers the expected scenario when the compactification is removed. As far as we know, this simple and expected connection between the parametric representation in the compactified scenario $(D, d)$ and the noncompactified scenario $(D)$ has never been explicitly stated.

In the following, we change the parametrization and proceed to show that the usual sum over the Bessel-K functions appears naturally. This is expected when dealing with Feynman amplitudes in periodically compactified spaces as shown in previous works $[18,29,56]$ restricted to contributions of Feynman graphs of low orders.

\section{A. Change of parametrization}

We employ a new parametrization that sectorizes the $u_{i}$-parameters such that there is one parameter $(s)$ that runs over the positive real axis and all others $\left(t_{i}\right)$ just lie in the interval $[0,1]$. This is done by the sectorization 


$$
\begin{aligned}
u_{1} & =s t_{1} \ldots t_{I-1}, \\
u_{2} & =s t_{1} \ldots t_{I-2}\left(1-t_{I-1}\right), \\
u_{3} & =s t_{1} \ldots t_{I-3}\left(1-t_{I-2}\right), \\
\vdots & =\vdots \\
u_{I-1} & =s t_{1}\left(1-t_{2}\right), \\
u_{I} & =s\left(1-t_{1}\right) .
\end{aligned}
$$

This change of variables produces $\prod_{i=1}^{I} d u_{i}=s^{I-1} d s \times$ $\prod_{i=1}^{I-1} t_{i}^{I-1-i} d t_{i}$. And, as all $u_{i}$ have a factor $s$, this will factor out in a way that allows us to define

$$
\begin{aligned}
U\left(u_{i}\right) & =s^{L} \bar{U}\left(t_{i}\right), \\
V\left(P ; u_{i}\right) & =s^{L+1} \bar{V}\left(P ; t_{i}\right), \\
A\left(u_{i}\right) & =s^{L-1} \bar{A}\left(t_{i}\right), \\
\tilde{B}\left(u_{i}\right) & =s \overline{\widetilde{B}}\left(t_{i}\right) .
\end{aligned}
$$

Notice that one must determine the new expressions by direct inspection.
With this notation, the amplitude related to the diagram becomes

$$
\begin{aligned}
& \mathcal{I}_{G}=C_{G} \int_{0}^{\infty} d s s^{I-1}\left[\prod_{i=1}^{I-1} \int_{0}^{1} d t_{i} t_{i}^{I-1-i}\right] \frac{e^{-s \mathcal{\mathcal { M } ^ { 2 }}\left(t_{i}, m_{i}\right)}}{(4 \pi)^{\frac{D}{2} L}} \frac{e^{-s \frac{\bar{\Sigma}\left(P_{i} t\right)}{U(t)}}}{s^{\frac{D L}{2}} \bar{U}^{\frac{D}{2}}} \\
& \times \sum_{\substack{n_{\alpha}^{(\ell)} \in \mathbb{Z} \\
\forall \ell, \forall \alpha}} e^{-\frac{1}{s} \sum_{\alpha} \frac{L_{\alpha}^{2}}{\alpha \bar{U}(t)} \mathbf{n}_{\alpha}^{t} \bar{A}(t) \mathbf{n}_{\alpha}} e^{\frac{2 \pi i}{\bar{U}(t)} \sum_{\alpha} \mathbf{n}_{\alpha}^{t} \bar{A}(t) \tilde{\tilde{B}}_{\alpha}}
\end{aligned}
$$

with

$$
\mathcal{M}^{2}\left[t_{i}, m_{i}^{2}\right]=\sum_{i} \frac{u_{i}}{s} m_{i}^{2}
$$

When all masses are equal $\left(m_{i}=m\right)$ we get simply $\mathcal{M}^{2}=m^{2}$.

By inspection, we have two kinds of integrals regarding the parameter $s$. When all modes are zero $\left(n_{\alpha}^{(\ell)}=0\right)$ we have a gamma function, when any of them are different from zero we get the integral that defines the modified Bessel function of the second kind (Bessel-K), that is

$$
\begin{aligned}
\mathcal{I}_{G}= & C_{G}\left[\prod_{i=1}^{I-1} \int_{0}^{1} d t_{i} t_{i}^{I-1-i}\right] \frac{\Gamma\left(I-\frac{D L}{2}\right)}{(4 \pi)^{\frac{D}{2} L} \bar{U}^{\frac{D}{2}}} \frac{1}{\left[\mathcal{M}^{2}\left(t_{i}, m_{i}\right)+\frac{\bar{V}(P ; t)}{\bar{U}(t)}\right]^{I-\frac{D L}{2}}} \\
& +\frac{2 C_{G}}{(4 \pi)^{\frac{D}{2} L}}\left[\prod_{i=1}^{I-1} \int_{0}^{1} d t_{i} t_{i}^{I-1-i}\right] \sum_{\substack{n_{\alpha}^{(t)} \in \mathbb{Z} \\
\forall \ell, \forall \alpha}}^{\frac{2 \pi i}{\bar{U}^{(}(t)} \sum_{\alpha} \mathbf{n}_{\alpha}^{t} \bar{A}(t) \overline{\tilde{B}}_{\alpha}}\left(\frac{\sum_{\alpha} L_{\alpha}^{2} \mathbf{n}_{\alpha}^{t} \bar{A}(t) \mathbf{n}_{\alpha}}{4 \bar{U}(t)\left[\mathcal{M}^{2}\left(t_{i}, m_{i}\right)+\frac{\bar{V}(P ; t)}{\bar{U}(t)}\right]}\right)^{\frac{I-D L / 2}{2}} \\
& \times K_{I-\frac{D L}{2}}\left(\sqrt{\frac{\sum_{\alpha} L_{\alpha}^{2} \mathbf{n}_{\alpha}^{t} \bar{A}(t) \mathbf{n}_{\alpha}}{\bar{U}(t)}} \sqrt{\mathcal{M}^{2}\left(t_{i}, m_{i}\right)+\frac{\bar{V}(P ; t)}{\bar{U}(t)}}\right)
\end{aligned}
$$

Where $\sum^{\prime}$ represents that we do not sum at the point where all modes are zero.

This completes the analysis. Once again we remark that this expression is suitable for large $L_{\alpha}$. This is much more evident at this point. If we try to extract information from small $L_{\alpha}$ we approach the $\operatorname{limit}_{\lim _{z \rightarrow 0}} K_{\nu}(z)$, where the Bessel-K function diverges and one must be very careful with the treatment, as discussed in Refs. [76,77]. Also, as the length parameter $L_{\alpha}$ is reduced each term of the sum gets bigger and the convergence of the summations takes longer. This is the justification for why we also exhibit the scenario near the dimensional reduction in Sec. III.

In the following section, we show the needed terms $U, V$, $A$, and $B$ for some Feynman graphs.

\section{SOME EXAMPLES IN $\phi^{3}$}

In this section, we assume that our model is the simple toy model $g \phi^{3}$ and exhibit the relevant information to write the amplitudes of certain diagrams both in the near dimensional reduction representation, Sec. III, and the near bulk representation, Sec. IV, for systems living in periodically compactified topologies. The required information, for each diagram, are the two Symanzik polynomials ( $U$ and $V$ ) and some complementary information as the matrix $M$ [see Eq. (28b)], $A$ (recall that $A=M^{-1} \operatorname{det} M=U M^{-1}$ ), and the vector $B$ (see Eq. (28c)). In what follows one can obtain the Symanzik polynomials by any of the three equivalent expressions: Eq. (17), Eq. (18), or Eq. (35). 


\section{A. One loop}

As a first example we consider the fish diagram, Fig. 1, with external momenta $Q$. Due to the existence of only one loop, the matrix $M$ is reduced to a number. We define for convenience that $u_{12}=u_{1}+u_{2}$, and we follow this notation in all other examples.

$$
\begin{gathered}
U=u_{12}=s, \\
V / Q^{2}=u_{1} u_{2}=s^{2}\{t(1-t)\}, \\
M=u_{12}=s, \\
A=M^{-1} \operatorname{det} M=1,
\end{gathered}
$$

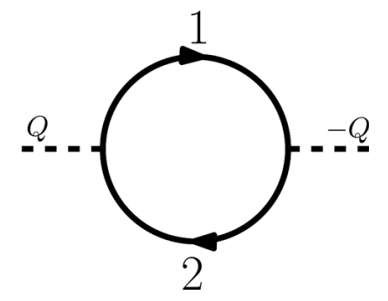

FIG. 1. Fish diagram, $\mathcal{I}_{1}$.

$$
\begin{aligned}
& B=u_{2} \omega_{\alpha}^{q}=s(1-t) \omega_{\alpha}^{q}, \\
& \tilde{Z}=n_{\alpha}^{q}(-t+\lfloor t\rceil) .
\end{aligned}
$$

In this simple scenario, the expression near bulk [Eq. (55)] is

$$
\begin{aligned}
\mathcal{I}_{1}= & C_{1} \int_{0}^{1} d t \frac{\Gamma\left(2-\frac{D}{2}\right)}{(4 \pi)^{\frac{D}{2}}} \frac{1}{\left[\mathcal{M}^{2}\left(t_{i}, m_{i}\right)+Q^{2} t(1-t)\right]^{2-\frac{D}{2}}}+\frac{2 C_{1}}{(4 \pi)^{\frac{D}{2}}} \int_{0}^{1} d t \sum_{\substack{n_{\alpha} \in \mathbb{Z} \\
\forall \alpha}}^{\prime} e^{2 \pi i \sum_{\alpha}(1-t) n_{\alpha} n_{\alpha}^{q}} \\
& \times\left(\frac{\sum_{\alpha} L_{\alpha}^{2} n_{\alpha}^{2}}{4\left[\mathcal{M}^{2}\left(t_{i}, m_{i}\right)+Q^{2} t(1-t)\right]}\right)^{\frac{2-D / 2}{2}} K_{2-\frac{D}{2}}\left(\sqrt{\sum_{\alpha} L_{\alpha}^{2} n_{\alpha}^{2}} \sqrt{\mathcal{M}^{2}\left(t_{i}, m_{i}\right)+Q^{2} t(1-t)}\right) .
\end{aligned}
$$

The second component is the finite-size contribution and the first component is the well-known bulk contribution, which is the dominant contribution,

$$
\left.\mathcal{I}_{1}\right|_{\text {bulk }} \sim \frac{C_{1} \Gamma\left(2-\frac{D}{2}\right)}{(4 \pi)^{\frac{D}{2}}} \int_{0}^{1} d t \frac{1}{\left[\mathcal{M}^{2}\left(t_{i}, m_{i}\right)+Q^{2} t(1-t)\right]^{2-\frac{D}{2}}}
$$

We could get a bit further and study, for example, the asymptotic behavior of this amplitude with respect to the external momenta $Q$. However, this falls outside the scope of this work and we shall return to this point in the near future.

The expression near dimensional reduction is [Eq. (47)]

$$
\left.\mathcal{I}_{1}\right|_{\text {dim .red. }}=\frac{C_{1} \Gamma\left(2-\frac{D-d}{2}\right)}{(4 \pi)^{\frac{D-d}{2}} \prod_{\alpha} L_{\alpha}} \int_{0}^{1} d t \sum_{\substack{n_{\alpha} \in \mathbb{Z} \\ \forall \alpha}} \frac{1}{\left[\mathcal{M}^{2}\left(t_{i}, m_{i}\right)+Q^{2} t(1-t)+\sum_{\alpha} \frac{4 \pi^{2}}{L_{\alpha}^{2}}\left(n_{\alpha}+n_{\alpha}^{q}(t-\lfloor t\rceil)\right)^{2}\right]^{2-\frac{D-d}{2}}},
$$

which produces, as the dominant contribution,

$$
\left.\mathcal{I}_{1}\right|_{\text {dim .red. }} \sim \frac{C_{1} \Gamma\left(2-\frac{D-d}{2}\right)}{(4 \pi)^{\frac{D-d}{2}} \prod_{\alpha} L_{\alpha}} \int_{0}^{1} d t \frac{1}{\left[\mathcal{M}^{2}\left(t_{i}, m_{i}\right)+Q^{2} t(1-t)+(t-\lfloor t\rceil)^{2} \sum_{\alpha}\left(\omega_{\alpha}^{q}\right)^{2}\right]^{2-\frac{D-d}{2}}} .
$$

Notice that the discussion from Sec. III becomes much more evident. If we compare Eq. (60) with Eq. (58) we see that the simple identification $D \rightarrow D-d$ (dimensionally reduced by hand) does not agree with the actual dimensional reduction. There is a surviving dependence related to the external modes $n_{\alpha}^{q}$ and the parameter $t$. However, if the external modes are null $\left(n_{\alpha}^{q}=0\right)$, the expression becomes what one would intuitively expect, that is, a simple identification $D \rightarrow D-d$ and $Q^{2} \rightarrow q^{2}$ (recall, once again, that $Q$ is the $D$-dimensional external momenta and $q$ is the $(D-d$ )-dimensional continuum external momenta). 
Let us take a 2-box $\left(\beta, L_{1}\right)$ scenario with $D=4, d=2$ and all internal masses as $m_{i}=m$,

$$
\left.\mathcal{I}_{1}\right|_{\text {dim.red. }} \sim \frac{C_{1}}{4 \pi \beta L_{1}} \int_{0}^{1} d t \frac{1}{m^{2}+Q^{2} t(1-t)+(t-\lfloor t\rceil)^{2}\left(\left(\omega_{\beta}^{q}\right)^{2}+\left(\omega_{L}^{q}\right)^{2}\right)} .
$$

To simplify notation let us use $\omega^{2}=\left(\left(\omega_{\beta}^{q}\right)^{2}+\left(\omega_{L}^{q}\right)^{2}\right)$ and, to make evident the contribution from the compactified and the noncompactified dimensions, let us write $Q^{2}=q^{2}+\omega^{2}$.

$$
\left.\mathcal{I}_{1}\right|_{\text {dim.red. }} \sim \frac{C_{1}}{4 \pi \beta L_{1}} \int_{0}^{1} d t \frac{1}{m^{2}+\left(q^{2}+\omega^{2}\right) t(1-t)+(t-\lfloor t\rceil)^{2} \omega^{2}} .
$$

We can split the domain at the point $t=1 / 2$. For $t<1 / 2$ we obtain $\lfloor t\rceil=0$, while for $t>1 / 2$ we get $\lfloor t\rceil=1$,

$$
\left.\mathcal{I}_{1}\right|_{\text {dim .red. }} \sim-\frac{C_{1}}{2 \beta L_{1} q^{2}} \int_{0}^{\frac{1}{2}} d t \frac{1}{-\frac{m^{2}}{q^{2}}-\frac{\left(q^{2}+\omega^{2}\right)}{q^{2}} t+t^{2}}=-\frac{C_{1}}{2 \beta L_{1} q^{2}} \int_{0}^{\frac{1}{2}} \frac{d t}{\chi_{+}-\chi_{-}}\left[\frac{1}{t-\chi_{+}}-\frac{1}{t-\chi_{-}}\right],
$$

with $\chi_{ \pm}$given by

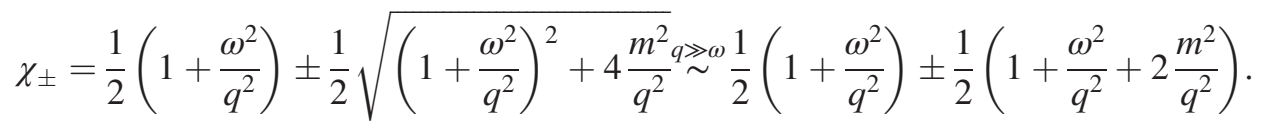

For large values of the continuum $D-d$ external momenta $q$ we obtain

$$
\left.\mathcal{I}_{1}\right|_{\text {dim .red. }} \sim-\left.\frac{C_{1}}{2 \beta L_{1}} \frac{1}{q^{2}+\omega^{2}+2 m^{2}} \ln \frac{t-\chi_{+}}{t-\chi_{-}}\right|_{\varepsilon} ^{\frac{1}{2}} \sim-\frac{C_{1}}{2 \beta L_{1}} \frac{1}{q^{2}+\omega^{2}+2 m^{2}} \ln \frac{q^{2}+2 \omega^{2}+2 m^{2}}{q^{2}+2 m^{2}} \frac{\varepsilon q^{2}+m^{2}}{q^{2}+\omega^{2}+m^{2}} .
$$

In the massive scenario ( $m \neq 0$ so we can take $\varepsilon=0)$ it reduces to

$$
\left.\mathcal{I}_{1}\right|_{\text {dim .red. }} \sim \frac{C_{1}}{2 \beta L_{1}} \frac{1}{q^{2}+\omega^{2}+2 m^{2}} \ln \frac{q^{4}+q^{2}\left(\omega^{2}+3 m^{2}\right)+2 m^{2}\left(\omega^{2}+m^{2}\right)}{\left(q^{2}+2 \omega^{2}+2 m^{2}\right) m^{2}} \sim \frac{C_{1}}{2 \beta L_{1}} \frac{\ln \left(q^{2}+\omega^{2}+3 m^{2}\right)}{q^{2}+\omega^{2}+2 m^{2}} .
$$

On the other hand, if one uses a "naive" dimensional reduction the contribution from $\omega^{2}$ does not exist. The difference is small, it might act as a small dynamic shift in the mass parameter as discussed in Sec. III.

In the massless scenario $(m=0, \varepsilon \neq 0)$ it becomes more evident that this contribution acts as an effective mass:

$$
\begin{aligned}
\left.\mathcal{I}_{1}\right|_{\text {dim .red. }} & \sim-\frac{C_{1}}{2 \beta L_{1}} \frac{1}{q^{2}+\omega^{2}} \ln \varepsilon \frac{q^{2}+2 \omega^{2}}{q^{2}+\omega^{2}}=-\frac{C_{1}}{2 \beta L_{1}} \frac{1}{q^{2}+\omega^{2}}\left[\ln \varepsilon+\ln \left(1+\frac{\omega^{2}}{q^{2}+\omega^{2}}\right)\right] \\
& \sim-\frac{C_{1}}{2 \beta L_{1}} \frac{1}{q^{2}+\omega^{2}}\left[\ln \varepsilon+\frac{\omega^{2}}{q^{2}+\omega^{2}}\right] .
\end{aligned}
$$

\section{B. Two loops}

With regard to mass corrections there are two one-particle irreducible (1P1) diagrams at two loops (see Fig. 2 and Fig. 3). For the first diagram, Fig. 2, the functions that allow us to reproduce both the near dimensional reduction and near bulk scenario are exhibited in Eq. (67).

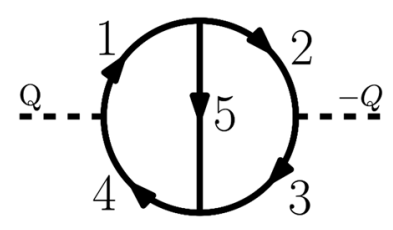

FIG. 2. 1PI diagrams at two loops, $\mathcal{I}_{2 a}$.

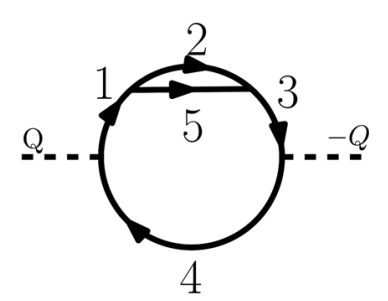

FIG. 3. 1PI diagrams at two loops, $\mathcal{I}_{2 b}$. 


$$
\begin{aligned}
& U=u_{5} u_{1234}+u_{14} u_{23}=s^{2}\left\{t_{1}\left[1-t_{1}+t_{1} t_{2}\left(1-t_{3} t_{4}-t_{2}\left(1-t_{3} t_{4}\right)^{2}\right)\right]\right\}, \\
& V / Q^{2}=u_{5} u_{12} u_{34}+u_{12} u_{3} u_{4}+u_{1} u_{2} u_{34} \\
& =s^{3}\left\{t_{1} t_{2}^{2} t_{3}\left[1-t_{1}-t_{2} t_{3}+t_{1} t_{2} t_{3}+t_{1} t_{4}\left(1-t_{2}\right)\left(1-t_{3} t_{4}\right)+t_{1}^{2}\left(1-t_{4}\right)\left(1-t_{3}\right)\left(1-t_{2}\left(1-t_{3} t_{4}\right)\right)\right]\right\}, \\
& M=\left(\begin{array}{cc}
u_{145} & -u_{5} \\
-u_{5} & u_{235}
\end{array}\right)=s\left(\begin{array}{cc}
1-t_{2}+t_{1} t_{2} t_{3} t_{4} & -1+t_{1} \\
-1+t_{1} & 1-t_{1}+t_{2}\left(1-t_{3} t_{4}\right)
\end{array}\right), \\
& A=M^{-1} \operatorname{det} M=\left(\begin{array}{cc}
u_{235} & u_{5} \\
u_{5} & u_{145}
\end{array}\right)=s\left(\begin{array}{cc}
1-t_{1}+t_{2}\left(1-t_{3} t_{4}\right) & 1-t_{1} \\
1-t_{1} & 1-t_{2}+t_{1} t_{2} t_{3} t_{4}
\end{array}\right) \\
& B_{\alpha}=\omega_{\alpha}^{q}\left(\begin{array}{l}
u_{4} \\
u_{3}
\end{array}\right)=s t_{1} \omega_{\alpha}^{q}\left(\begin{array}{c}
1-t_{2} \\
t_{2}\left(1-t_{3}\right)
\end{array}\right)
\end{aligned}
$$

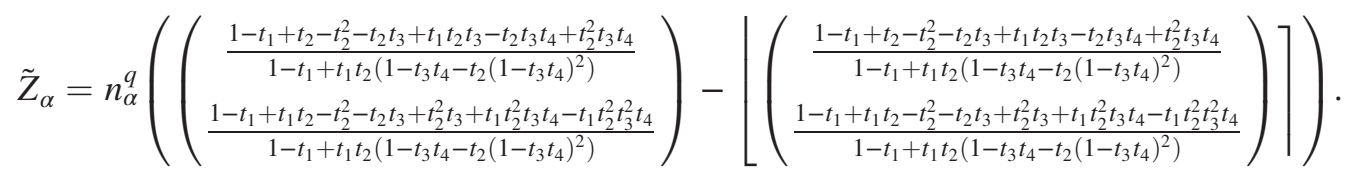

With the expressions in Eq. (67) we can write the expression both for the large-box and small-box scenarios. We exhibit only the dominant contribution in each case. To obtain the dominant contribution near the bulk we start with Eq. (55), take only the zeroth internal mode, and substitute the expressions in Eq. (67),

$$
\begin{aligned}
\left.\mathcal{I}_{2 a}\right|_{\text {bulk }} \sim & \frac{C_{2 a} \Gamma(5-D)}{(4 \pi)^{D}} \int_{0}^{1} \frac{d t_{1} d t_{2} d t_{3} d t_{4} t_{1}^{3} t_{2}^{2} t_{3}}{\left\{t_{1}\left[1-t_{1}+t_{1} t_{2}\left(1-t_{3} t_{4}-t_{2}\left(1-t_{3} t_{4}\right)^{2}\right)\right]\right\}^{\frac{D}{2}}} \\
& \times\left[\mathcal{M}^{2}\left(t_{i}, m_{i}\right)+Q^{2} \frac{\left.t_{2}^{2} t_{3}\left[1-t_{1}-t_{2} t_{3}+t_{1} t_{2} t_{3}+t_{1} t_{4}\left(1-t_{2}\right)\left(1-t_{3} t_{4}\right)+t_{1}^{2}\left(1-t_{4}\right)\left(1-t_{3}\right)\left(1-t_{2}\left(1-t_{3} t_{4}\right)\right)\right]\right]^{D-5}}{1-t_{1}+t_{1} t_{2}\left(1-t_{3} t_{4}-t_{2}\left(1-t_{3} t_{4}\right)^{2}\right)} .\right.
\end{aligned}
$$

To obtain the dominant contribution near the dimensional reduction we start from Eq. (47). Just like the previous case at one loop the expression in the scenario of dimensional reduction is almost the same as in the bulk [taking the identification $D \rightarrow D-d$, adding the multiplicative factor $\left.\left(\prod_{\alpha} L_{\alpha}\right)^{L}\right]$ but with new dependence on the external modes $n_{\alpha}^{q}$ and the parameters $t_{i}$, which gives

$$
\begin{aligned}
\left.\mathcal{I}_{2 a}\right|_{\text {dim.red. }} \sim & \frac{C_{2 a} \Gamma(5+d-D)}{(4 \pi)^{D-d}\left(\prod_{\alpha} L_{\alpha}\right)^{L}} \int_{0}^{1} \frac{d t_{1} d t_{2} d t_{3} d t_{4} t_{1}^{3} t_{2}^{2} t_{3}}{\left\{t_{1}\left[1-t_{1}+t_{1} t_{2}\left(1-t_{3} t_{4}-t_{2}\left(1-t_{3} t_{4}\right)^{2}\right)\right]\right\}^{\frac{D-d}{2}}}\left[\mathcal{M}^{2}\left(t_{i}, m_{i}\right)+\bar{Z}_{\alpha}^{t} \bar{M} \bar{Z}_{\alpha}\right. \\
& \left.+Q^{2} \frac{t_{2}^{2} t_{3}\left[1-t_{1}-t_{2} t_{3}+t_{1} t_{2} t_{3}+t_{1} t_{4}\left(1-t_{2}\right)\left(1-t_{3} t_{4}\right)+t_{1}^{2}\left(1-t_{4}\right)\left(1-t_{3}\right)\left(1-t_{2}\left(1-t_{3} t_{4}\right)\right)\right]}{1-t_{1}+t_{1} t_{2}\left(1-t_{3} t_{4}-t_{2}\left(1-t_{3} t_{4}\right)^{2}\right)}\right]^{D-d-5} .
\end{aligned}
$$

Of course, the manipulation of this equation becomes much more intricate than the previous one-loop scenario.

For the second 1PI diagram, in Fig. 3, the two scenarios are reproduced if we use the functions in Eq. (70).

$$
\begin{aligned}
U & =u_{5} u_{2}+u_{134} u_{25}=s^{2} t_{1}\left\{\left[1-t_{2} t_{3}\left(1-t_{4}\right)\right]\left[1-t_{1}+t_{1} t_{2} t_{3}\left(1-t_{4}\right)\right]+t_{2} t_{3}\left(1-t_{1}\right)\left(1-t_{4}\right)\right\}, \\
V / Q^{2} & =u_{4} u_{2} u_{5}+u_{4} u_{13} u_{25}, \\
& =s^{3} t_{1}^{2} t_{2}\left\{t_{3}\left(1-t_{1}\right)\left(1-t_{2}\right)\left(1-t_{4}\right)+\left(1-t_{2}\right)\left(1-t_{3}+t_{3} t_{4}\right)\left(1-t_{1}+t_{1} t_{2} t_{3}\left(1-t_{4}\right)\right)\right\}, \\
M & =\left(\begin{array}{cc}
u_{1345} & -u_{5} \\
-u_{5} & u_{25}
\end{array}\right)=s\left(\begin{array}{cc}
1-t_{1} t_{2} t_{3}+t_{1} t_{2} t_{3} t_{4} & -1+t_{1} \\
-1+t_{1} & 1-t_{1}+t_{1} t_{2} t_{3}\left(1-t_{4}\right)
\end{array}\right),
\end{aligned}
$$




$$
\begin{aligned}
A & =M^{-1} \operatorname{det} M=\left(\begin{array}{cc}
u_{25} & u_{5} \\
u_{5} & u_{1345}
\end{array}\right)=s\left(\begin{array}{cc}
1-t_{1}+t_{1} t_{2} t_{3}\left(1-t_{4}\right) & 1-t_{1} \\
1-t_{1} & 1-t_{1} t_{2} t_{3}+t_{1} t_{2} t_{3} t_{4}
\end{array}\right), \\
B_{\alpha}= & \omega_{\alpha}^{q}\left(\begin{array}{c}
u_{4} \\
0
\end{array}\right)=s t_{1} \omega_{\alpha}^{q}\left(\begin{array}{c}
1-t_{2} \\
0
\end{array}\right), \\
\tilde{Z}_{\alpha}= & \omega_{\alpha}^{q}\left(\frac{\left(\begin{array}{c}
\left(1-t_{1}\right)\left(1-t_{2}\right)+t_{1} t_{2} t_{3}\left(1-t_{2}\right)\left(1-t_{4}\right) \\
\left(1-t_{1}\right)\left(1-t_{2}\right)
\end{array}\right)}{\left[1-t_{2} t_{3}\left(1-t_{4}\right)\right]\left[1-t_{1}+t_{1} t_{2} t_{3}\left(1-t_{4}\right)\right]+t_{2} t_{3}\left(1-t_{1}\right)\left(1-t_{4}\right)}\right. \\
& \left.-\left\lfloor\frac{\left(\begin{array}{c}
\left(1-t_{1}\right)\left(1-t_{2}\right)+t_{1} t_{2} t_{3}\left(1-t_{2}\right)\left(1-t_{4}\right) \\
\left(1-t_{1}\right)\left(1-t_{2}\right)
\end{array}\right)}{\left[1-t_{2} t_{3}\left(1-t_{4}\right)\right]\left[1-t_{1}+t_{1} t_{2} t_{3}\left(1-t_{4}\right)\right]+t_{2} t_{3}\left(1-t_{1}\right)\left(1-t_{4}\right)}\right]\right) .
\end{aligned}
$$

\section{Three loops}

At three loops we have a large number of 1PI diagrams that contribute to the correction of the full propagator. One such example is shown in Fig. 4 and the expression for the Symanzik polynomials and the matrices and vector needed are shown in Eq. (71). One can use directly the matrices $A$ and the vector $B$ to build the representation near the bulk [see Eq. (55)] and use $M$ to build the representation near the dimensional reduction [see Eq. (47)].

$$
U=u_{34} u_{56} u_{1278}+u_{34} u_{18} u_{27}+u_{3} u_{4} u_{1278}
$$

$$
\begin{aligned}
V / Q^{2}= & u_{12} u_{34} u_{56} u_{78}+u_{1} u_{2} u_{34} u_{78} \\
& +u_{12} u_{3} u_{4} u_{78}+u_{12} u_{34} u_{7} u_{8},
\end{aligned}
$$

$$
M=\left(\begin{array}{ccc}
u_{14568} & -u_{456} & u_{4} \\
-u_{456} & u_{24567} & -u_{4} \\
u_{4} & -u_{4} & u_{34}
\end{array}\right)
$$

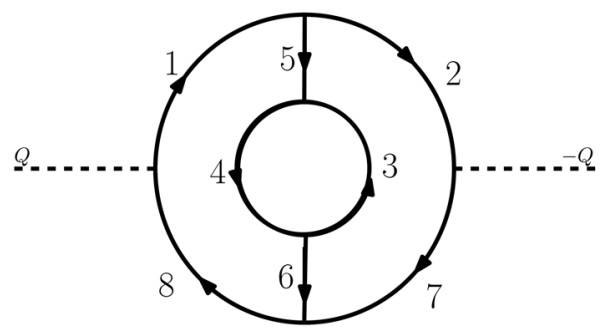

FIG. 4. Example with three loops, $\mathcal{I}_{3}$.

$$
A=M^{-1} \operatorname{det} M=\left(\begin{array}{ccc}
u_{2567} u_{34} & u_{3} u_{4} & -u_{4} u_{27} \\
+u_{3} u_{4} & +u_{34} u_{56} & \\
u_{3} u_{4} & u_{1568} u_{34} & \\
+u_{34} u_{56} & +u_{3} u_{4} & u_{4} u_{18} \\
-u_{4} u_{27} & u_{4} u_{18} & u_{456} u_{1278} \\
& & +u_{18} u_{27}
\end{array}\right) \text {, }
$$

$$
B_{\alpha}=\omega_{\alpha}^{q}\left(\begin{array}{c}
u_{8} \\
u_{7} \\
0
\end{array}\right) .
$$

\section{CONCLUSION}

We manage to show two useful parametric representations for Feynman diagrams in periodically compactified spaces. One representation is more suitable in a large-box regime and recovers the expected standard Schwinger parametric representation for noncompactified theories at the bulk. The other representation is more suitable in a small-box regime and shows how the amplitudes behave if we consider the limit of a dimensional reduction. Both expressions allow one to obtain the behavior with respect to the compactification length and shall be helpful for those interested in higher-order Feynman diagrams in compactified spaces to study a myriad of phenomena as depicted by works in quantum field theory with compactifications [14,18,26-29,56,57].

Of course, we still do not get the full picture and there are many remaining tasks to be done: 1) There is a need to extend the present work for fields with nonzero spins; 2) It might be useful to consider scenarios where space is compactified by a different prescription other than a periodic one (Dirichlet, Neumann, Robin, ...); 3) It is of 
utmost importance to extract asymptotic information from the parametric representations so we can easily understand the behavior of a higher-order graph in compactified space. Each of these topics are the subject of future works.

\section{ACKNOWLEDGMENTS}

The author thanks the Brazilian agency Conselho Nacional de Desenvolvimento Científico e Tecnológico (CNPq) for financial support.
[1] J. Zinn-Justin, Quantum Field Theory and Critical Phenomena, 4th ed. (Clarendon Press, Oxford, 2002).

[2] C. Itzykson and J. Zuber, Quantum Field Theory, International Series In Pure and Applied Physics (McGraw-Hill, New York, 1980).

[3] V. Rivasseau, From Perturbative to Constructive Renormalization (Princton University Press, New Jersey, 2014).

[4] R. B. Paris and D. Kaminski, Asymptotics and MellinBarnes Integrals, Encyclopedia of Mathematics and its Applications Vol. 85 (Cambridge University Press, Cambridge, England, 2001).

[5] V. A. Smirnov, Analytic tools for Feynman integrals, Springer Tracts in Modern Physics Vol. 250 (Springer, Berlin, Heidelberg, 2012).

[6] C. de Calan and A. P. C. Malbouisson, Complete Mellin representation and asymptotic behaviors of Feynman amplitudes, Ann. Inst. Henri Poincaré Phys. Theor. 32, 91 (1980), http://www.numdam.org/item/?id=AIHPA_1980_ 32_1_91_0.

[7] C. de Calan, F. David, and V. Rivasseau, Renormalization in the complete Mellin representation of Feynman amplitudes, Commun. Math. Phys. 78, 531 (1981).

[8] A. P. C. Malbouisson, A convergence theorem for asymptotic expansions of Feynman amplitudes, J. Phys. A 33, 3587 (2000).

[9] C. A. Linhares, A. P. C. Malbouisson, and I. Roditi, Asymptotic expansions of feynman amplitudes in a generic covariant gauge, Int. J. Mod. Phys. A 23, 1089 (2008).

[10] R. Gurau, A. P. C. Malbouisson, V. Rivasseau, and A. Tanasa, Non-commutative complete Mellin representation for Feynman amplitudes, Lett. Math. Phys. 81, 161 (2007).

[11] R. Gurau, V. Rivasseau, and A. Sfondrini, Renormalization: An advanced overview, arXiv:1401.5003.

[12] T. Appelquist and R. D. Pisarski, High-temperature YangMills theories and three-dimensional quantum chromodynamics, Phys. Rev. D 23, 2305 (1981).

[13] N. P. Landsman and C. G. van Weert, Real and imaginary time field theory at finite temperature and density, Phys. Rep. 145, 141 (1987).

[14] N. P. Landsman, Limitations to dimensional reduction at high temperature, Nucl. Phys. B322, 498 (1989).

[15] M. Le Bellac, Quantum and Statistical Field Theory (Clarendon, Oxford, UK, 1991), p. 592.

[16] A. K. Das, Finite Temperature Field Theory (World Scientific, New York, 1997).

[17] J. I. Kapusta and C. Gale, Finite-temperature Field Theory: Principles and Applications, Cambridge Monographs on Mathematical Physics (Cambridge University Press, Cambridge, England, 2011).

[18] F. C. Khanna, A. P. C. Malbouisson, J. M. C. Malbouisson, and A. R. Santana, Thermal Quantum Field TheoryAlgebraic Aspects and Applications (World Scientific Publishing Company, Singapore, 2009).

[19] Y. Tanizaki, T. Misumi, and N. Sakai, Circle compactification and 't Hooft anomaly, J. High Energy Phys. 12 (2017) 056.

[20] J. E. Hetrick and Y. Hosotani, Yang-Mills theory on a circle, Phys. Lett. B 230, 88 (1989).

[21] K. Aitken, A. Cherman, E. Poppitz, and L. G. Yaffe, QCD on a small circle, Phys. Rev. D 96, 096022 (2017).

[22] M. Nitta, Fractional instantons and bions in the principal chiral model on $\mathbb{R}^{2} \times \mathbb{S}^{1}$ with twisted boundary conditions, J. High Energy Phys. 08 (2015) 063.

[23] T. Kanazawa, M. Ünsal, and N. Yamamoto, Phases of circlecompactified QCD with adjoint fermions at finite density, Phys. Rev. D 96, 034022 (2017).

[24] E. Witten, Constraints on supersymmetry breaking, Nucl. Phys. B202, 253 (1982).

[25] G. Plunien, B. Muller, and W. Greiner, The Casimir effect, Phys. Rep. 134, 87 (1986).

[26] M. Bordag, U. Mohideen, and V. M. Mostepanenko, New developments in the Casimir effect, Phys. Rep. 353, 1 (2001).

[27] K. A. Milton, The Casimir effect: Recent controversies and progress, J. Phys. A 37, R209 (2004).

[28] G. L. Klimchitskaya, U. Mohideen, and V. M. Mostepanenko, The Casimir force between real materials: Experiment and theory, Rev. Mod. Phys. 81, 1827 (2009).

[29] E. Elizalde, Ten Physical Applications of Spectral Zeta Functions (Springer, Heidelberg, Germany, 2012), Vol. 855.

[30] J. H. Schwarz, Superstring theory, Phys. Rep. 89, 223 (1982).

[31] P. Horava and E. Witten, Eleven-dimensional supergravity on a manifold with boundary, Nucl. Phys. B475, 94 (1996).

[32] O. Aharony, S. S. Gubser, J. M. Maldacena, H. Ooguri, and Y. Oz, Large $\mathrm{N}$ field theories, string theory and gravity, Phys. Rep. 323, 183 (2000).

[33] D. Chakraverty, K. Huitu, and A. Kundu, Effects of universal extra dimensions on B0-antiB0 mixing, Phys. Lett. B 558, 173 (2003).

[34] K. Agashe, G. Perez, and A. Soni, B-Factory Signals for a Warped Extra Dimension, Phys. Rev. Lett. 93, 201804 (2004).

[35] L. Da Rold and A. Pomarol, Chiral symmetry breaking from five dimensional spaces, Nucl. Phys. B721, 79 (2005). 
[36] G. Panico, M. Serone, and A. Wulzer, A model of electroweak symmetry breaking from a fifth dimension, Nucl. Phys. B739, 186 (2006).

[37] G. Panico, M. Serone, and A. Wulzer, Electroweak symmetry breaking and precision tests with a fifth dimension, Nucl. Phys. B762, 189 (2007).

[38] M. Blanke, A. J. Buras, B. Duling, K. Gemmler, and S. Gori, Rare $K$ and $B$ decays in a warped extra dimension with custodial protection, J. High Energy Phys. 03 (2009) 108.

[39] C. C. Ttira, C. D. Fosco, A. P. C. Malbouisson, and I. Roditi, Vacuum polarization for compactified $\mathrm{QED}_{4+1}$ in a magnetic flux background, Phys. Rev. A 81, 032116 (2010).

[40] L. P. R. Ospedal and J. A. Helayël-Neto, Fermion interparticle potentials in $5 \mathrm{D}$ and a dimensional restriction prescription to 4D, Phys. Rev. D 97, 056014 (2018).

[41] L. Bhardwaj, P. Jefferson, H.-C. Kim, H.-C. Tarazi, and C. Vafa, Twisted circle compactifications of 6d SCFTs, J. High Energy Phys. 12 (2020) 151.

[42] J. Gasser and H. Leutwyler, Spontaneously broken symmetries: Effective Lagrangians at finite volume, Nucl. Phys. B307, 763 (1988).

[43] C. J. D. Lin, G. Martinelli, C. T. Sachrajda, and M. Testa, $\mathrm{K}->$ pi pi decays in a finite volume, Nucl. Phys. B619, 467 (2001).

[44] G. Colangelo, S. Durr, and C. Haefeli, Finite volume effects for meson masses and decay constants, Nucl. Phys. B721, 136 (2005).

[45] S. Aoki, H. Fukaya, S. Hashimoto, and T. Onogi, Finite volume QCD at fixed topological charge, Phys. Rev. D 76, 054508 (2007).

[46] M. Hayakawa and S. Uno, QED in finite volume and finite size scaling effect on electromagnetic properties of hadrons, Prog. Theor. Phys. 120, 413 (2008).

[47] L. F. Palhares, E. S. Fraga, and T. Kodama, Chiral transition in a finite system and possible use of finite size scaling in relativistic heavy ion collisions, J. Phys. G 38, 085101 (2011).

[48] V. Bernard, M. Lage, U. G. Meissner, and A. Rusetsky, Scalar mesons in a finite volume, J. High Energy Phys. 01 (2011) 019.

[49] R. A. Briceno and Z. Davoudi, Moving multichannel systems in a finite volume with application to proton-proton fusion, Phys. Rev. D 88, 094507 (2013).

[50] A. Bhattacharyya, P. Deb, S. K. Ghosh, R. Ray, and S. Sur, Thermodynamic properties of strongly interacting matter in finite volume using Polyakov-Nambu-Jona-Lasinio model, Phys. Rev. D 87, 054009 (2013).

[51] M. G. Perez, A. Gonzalez-Arroyo, and M. Okawa, Volume independence for Yang-Mills fields on the twisted torus, Int. J. Mod. Phys. A 29, 1445001 (2014).

[52] A. Bhattacharyya, R. Ray, and S. Sur, Fluctuation of strongly interacting matter in the Polyakov-Nambu-JonaLasinio model in a finite volume, Phys. Rev. D 91, 051501 (2015).

[53] A. Bhattacharyya, S. K. Ghosh, R. Ray, K. Saha, and S. Upadhaya, Polyakov-Nambu-Jona-Lasinio model in finite volumes, Europhys. Lett. 116, 52001 (2016).

[54] A. Juricic and B.-J. Schaefer, Chiral thermodynamics in a finite box, Acta Phys. Pol. B Proc. Suppl. 10, 609 (2017).
[55] B.-L. Li, Z.-F. Cui, B.-W. Zhou, S. An, L.-P. Zhang, and H.-S. Zong, Finite volume effects on the chiral phase transition from Dyson-Schwinger equations of QCD, Nucl. Phys. B938, 298 (2019).

[56] F. C. Khanna, A. P. C. Malbouisson, J. M. C. Malbouisson, and A.E. Santana, Quantum field theory on toroidal topology: Algebraic structure and applications, Phys. Rep. 539, 135 (2014).

[57] S. Mogliacci, I. Kolbé, and W. A. Horowitz, Geometrically confined thermal field theory: Finite size corrections and phase transitions, Phys. Rev. D 102, 116017 (2020).

[58] E. Cavalcanti, C. A. Linhares, and A. P. C. Malbouisson, Properties of size-dependent models having quasiperiodic boundary conditions, Int. J. Mod. Phys. A 33, 1850008 (2018).

[59] L. M. Abreu, A. P. C. Malbouisson, J. M. C. Malbouisson, and A.E. Santana, Large $N$ transition temperature for superconducting films in a magnetic field, Phys. Rev. B 67, 212502 (2003).

[60] L. M. Abreu, C. de Calan, A. P. C. Malbouisson, J. M. C. Malbouisson, and A. E. Santana, Critical behaviour of the compactified lambda phi**4 theory, J. Math. Phys. (N.Y.) 46, 012304 (2005).

[61] C. A. Linhares, A. P. C. Malbouisson, Y. W. Milla, and I. Roditi, First-order phase transitions in superconducting films: A Euclidean model, Phys. Rev. B 73, 214525 (2006).

[62] L. M. Abreu, A. P. C. Malbouisson, J. M. C. Malbouisson, and A. E. Santana, Finite-size effects on the chiral phase diagram of four-fermion models in four dimensions, Nucl. Phys. B819, 127 (2009).

[63] L. M. Abreu, A. P. C. Malbouisson, and J. M. C. Malbouisson, Nambu-Jona-Lasinio model in a magnetic background: Sizedependent effects, Phys. Rev. D 84, 065036 (2011).

[64] L. M. Abreu, A. P. C. Malbouisson, and J. M. C. Malbouisson, Finite-size effects on the phase diagram of difermion condensates in two-dimensional four-fermion interaction models, Phys. Rev. D 83, 025001 (2011).

[65] C. A. Linhares, A. P. C. Malbouisson, and M. L. Souza, A note on the infrared behavior of the compactified GinzburgLandau model in a magnetic field, Europhys. Lett. 96, 31002 (2011).

[66] F. C. Khanna, A. P. C. Malbouisson, J. M. C. Malbouisson, and A. E. Santana, Finite-size effects on the phase transition in the three-dimensional Gross-Neveu model, Europhys. Lett. 97, 11002 (2012).

[67] F. C. Khanna, A. P. C. Malbouisson, J. M. C. Malbouisson, and A. E. Santana, Phase transition in the massive GrossNeveu model in toroidal topologies, Phys. Rev. D 85, 085015 (2012).

[68] C. A. Linhares, A. P. C. Malbouisson, J. M. C. Malbouisson, and I. Roditi, Spontaneous symmetry restoration in a field theory at finite chemical potential in a toroidal topology, Phys. Rev. D 86, 105022 (2012).

[69] L. M. Abreu, C. A. Linhares, A. P. C. Malbouisson, and J. M.C. Malbouisson, Magnetic effects on spontaneous symmetry breaking/restoration in a toroidal topology, Phys. Rev. D 88, 107701 (2013).

[70] E. B. S. Corrêa, C. A. Linhares, and A. P. C. Malbouisson, Finite-size, magnetic and chemical-potential effects on firstorder phase transitions, Phys. Lett. A 377, 1984 (2013). 
[71] E. Cavalcanti, E. Castro, C. A. Linhares, and A. P. C. Malbouisson, Influence of external magnetic field, finitesize effects and chemical potential on the phase transition of a complex scalar field, Eur. Phys. J. C 77, 711 (2017).

[72] M. Benhamou and A. Kassou-ou Ali, Schwinger alpha parametric representation of finite temperature field theories: Renormalization, Int. J. Mod. Phys. A 05, 4427 (1990).

[73] M. Benhamou and A. Kassou-ou Ali, The schwinger $\alpha$-parametric representation of the finite-temperature field theory: Renormalization II, Int. J. Mod. Phys. A 07, 193 (1992).

[74] F. C. Khanna, A. P. C. Malbouisson, J. M. C. Malbouisson, and A. E. Santana, Dimensional renormalizability in compactified spaces, arXiv:0906.5080.

[75] F. L. Cardoso and C. A. Linhares (to be published).

[76] E. Cavalcanti, J. A. Lourenço, C. A. Linhares, and A. P. C. Malbouisson, Dimensional reduction of a finite-size scalar field model at finite temperature, Phys. Rev. D 99, 025007 (2019).

[77] E. Cavalcanti, C. Linhares, J. Lourenço, and A. Malbouisson, Effect of boundary conditions on dimensionally reduced field-theoretical models at finite temperature, Phys. Rev. D 100, 025008 (2019).

[78] I. T. Todorov, Analytic Properties of Feynman Diagrams in Quantum Field Theory, International Series of Monographs in Natural Philosophy Vol. 38 (Pergamon, New York, 1971).

[79] M. E. Fisher, Critical phenomena in films and surfaces, J. Vac. Sci. Technol. 10, 665 (1973).

[80] S. Datta and S. Gupta, Dimensional reduction and screening masses in pure gauge theories at finite temperature, Nucl. Phys. B534, 392 (1998).

[81] B. Grossmann, S. Gupta, U. M. Heller, and F. Karsch, Glueball-like screening masses in pure su (3) at finite temperatures, Nucl. Phys. B417, 289 (1994). 